Chinese Journal of Organic Chemistry

\title{
統基-烯/炔点击化学研究进展
}

\author{
刘清张秋禹* 陈少杰周 健雷星锋 \\ (西北工业大学理学院应用化学系 教育部空间物理与化学重点实验室 西安 710129)
}

\begin{abstract}
摘要 近年来, 点击化学以其应用范围广、反应条件简单、速度快, 产率高、环境友好、选择性强等诸多优点受到科 学家的青睐, 点击化学的概念对有机合成领域有很大的贡献, 在药物开发和生物医用材料等诸多领域中, 它已经成为 目前最为有用和吸引人的合成理念之一. 而巯基一烯/炔点击化学是近年来发展衍生出来的一类新型的点击化学, 它以 光引发自由基反应为催化介质，充分将光引发过程的优点和传统的点击反应的特点相结合，在特定的区域和官能团间 反应, 具有高度的选择性, 成为合成材料的又一重要途径. 本文着重就疏基-烯/炔点击化学在制备功能性聚合物微球、 两亲性的嵌段聚合物、分子器件材料、高度支化聚合物等领域及化学修饰与改性方面进行了评述, 并对点击化学在新 领域中的应用及其发展方向进行了展望.
\end{abstract}

关键词 点击化学; 颈基-烯/炔; 功能性聚合物微球; 两亲性的嵌段聚合物; 分子器件材料; 高度支化聚合物; 化学修 饰与改性

\section{Progress in Thiol-Ene/Yne Click Chemistry}

\author{
Liu, Qing Zhang, Qiuyu* Chen, Shaojie Zhou, Jian $\quad$ Lei, Xingfeng \\ (Key Laboratory of Applied Physics and Chemistry of Ministry of Education, Department of Applied Chemistry, School of \\ Science, Northwestern Polytechnical University, Xi'an 710072)
}

\begin{abstract}
Click chemistry has attracted great interests due to a wide variety of superior merits, including a wide range of applications, simplified experimental condition, rapid reaction speed, higher functional group conversion, environmental friendly and selectivity. And conception of click chemistry contributed to the synthetic chemistry apparently, in particular, with respect to pharmaceutical manufacture and biomedical materials, resulting in the most useful and promising synthetic method. Furthermore, thiol-ene/yne click chemistry, utilizing inclusion of visible light or UV light as catalytic medium, is a comparatively novel kind of click chemistry in comparison to several types of conventional reactions. In addition, the properties of the creative reaction stems from the combination the photoinitiated chemical reaction with the forementioned traditional click chemistry, providing a flexible approach to occur reaction within the oriented region and functional group. This review highlights the recent research on the preparation of functional polymeric microsphere, amphipathic block well-defined polymer, materials employed in the molecular device, desired dendritic polymers and chemistry modification via thiol-ene/yne click chemistry. Moreover, the problems and solutions are given for the future development of thiol-ene/yne click chemistry.

Keywords click chemistry; thiol-ene/yne; functional polymeric microsphere; amphipathic block polymer; molecular device; dendritic polymers; surface modification
\end{abstract}

点击化学(Click Chemistry), 又译作 “链接化学” 、 “动态组合化学”, 是由化学家 Sharpless ${ }^{[1]}$ 在 2001 年引 入的一个有机合成概念, 主旨是通过小单元的拼接, 尤 其强调在温和的反应条件下高选择性的形成碳-杂原子 键 $(\mathrm{C}-\mathrm{X}-\mathrm{C})$, 快速可靠地完成形形色色分子的合成, 实现分子多样性构建. 在绿色合成和原子经济时代的背
景下，点击化学的提出为构建功能性分子系统开拓出一 条高通量、高产率、高选择性的合成路线 ${ }^{[2,3]}$.

点击化学反应具有以下共同特征: (1)应用 “组合” 的概念，应用范围广; (2)反应条件简单(在水中或良溶剂 中进行)、速度快, 产率高、副产物少、环境友好; (3)反 应具有很强的立体选择性; (4)反应原料和试剂易得; (5)

\footnotetext{
*E-mail: qyzhang@nwpu.edu.cn

Received January 14, 2012; revised April 13, 2012; accepted, May 18, 2012.

Project supported by the National Natural Science Foundation of China (No. 51173146/E030904) and the Major State Basic Research Development Program of China (973 Program) (No. 2010CB635111).

国家自然科学基金(No. 51173146/E030904)和国家重点基础研究发展规划(973 计划)(No. 2010CB635111)资助项目.
} 
符合原子经济. 点击化学的典型代表反应为铜催化的叠 氮一炔基 Husigen 环加成反应 CuAAC (copper-catalyzed azide-alkyne cycloaddition)和烯烃间的 Diels-Alder 点击 反应. 点击化学的概念对化学合成领域具有很大的贡 献, 如大分子工程 ${ }^{[4 \sim 8]}$ 、药物合成 ${ }^{[9 \sim 11]}$ 、蛋白质组

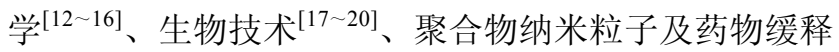

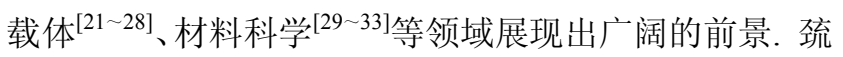
基-烯化学反应自发现以来一直备受关注, Jacobine ${ }^{[34]}$ 于 20 世纪 90 年代初系统的综述了不同类型颈基-烯化学 反应的反应机理及其应用研究, 指出了颈基-烯在光固 化胶粘剂和涂料领域的巨大应用前景; 21 世纪初, Hoyle 等 ${ }^{[35]}$ 总结了近十年的颈基-烯光化学反应的研究现状, 重点评述了颈基-烯光交联固化物的力学性能和物理化 学性质, 指出颈基一烯光交联反应在制备物理机械性能 可调的聚合物材料领域前景广阔. 近几年, Bowman 和 Holye 等 ${ }^{[36,37]}$ 指出颈基-烯/炔光化学反应是近年来发展 衍生出的一类新型的点击反应, 它以光引发自由基反应 为催化介质, 充分将光引发过程的优点和传统的点击反 应的优点相结合，在特定的区域和官能团间反应，具有 高度的选择性, 并可与多种化学合成方法交互使用, 如 颈基点击化学与控制聚合结合研究, 成为有机材料制备 的又一重要途径.

为此, 本文在前人研究統基-烯/炔点击化学的基础 上, 着重就颈基-烯/炔点击化学在制备功能性聚合物微 球、两亲性的嵌段聚合物、分子器件材料、高度支化聚 合物等领域及化学修饰与改性方面进行一一阐述.

\section{1 統基-烯/炔点击化学概述}

颈基-烯/炔的点击反应是继 Huisgen 反应之后又一 典型点击化学反应, 颈基-烯/炔的点击反应分为颈基烯点击反应体系和颈基-炔点击反应体系两种, 下文分 别就颈基-烯点击反应体系及颈基-炔点击反应体系进 行阐述.

颈基-烯点击反应最早由 Posner ${ }^{[38]}$ 于 1905 发现, 1938 年 Kharasch 等 ${ }^{[39]}$ 提出了颈基-烯光化学反应的反应 机理, 如图 1 所示. 第 1 步引发反应中, 引发剂吸收光子 裂解形成自由基, 自由基从附近的硫醇化合物的颈基夺 取一个氢, 硫醇转换为颈基自由基, 颈基自由基进攻富 电子双键发生自由基加成反应, 并在硫醇分子的参与下 完成颈基-烯光化学反应(步骤 2 和 3), 步骤 4 是典型的 聚合反应终止过程. Hoyle 等 ${ }^{[40]}$ 认为光引发的颈基和可 均聚型双键单体的光化学反应体系中是一个链增长机 理和逐步增长机理相互竞争的光聚合反应，而颈基和非 均聚型双键单体光化学反应体系中是单纯的逐步聚合 机理 ${ }^{[41,42]}$. 参与点击反应的双键单体反应活性顺序为降
冰片类和乙烯基醚最高, (甲基)丙烯酸类、丙烯腈类、苯 乙烯、马来酸类和共轭二烯逐步减弱. 颈基-烯点击反应 最早应用于制备结构规整的交联网络聚合物，并表现出 可调的的物理和化学性质, 本课题组 ${ }^{[43,44]}$ 以巯基-烯光 聚合体系快速制备具有可调的物理化学性质的交联网 络聚合物, 研究了双键单体和統基化合物结构和官能度 对颈基-烯光聚合过程动力学以及材料的热机械性能的 影响进行了的研究.

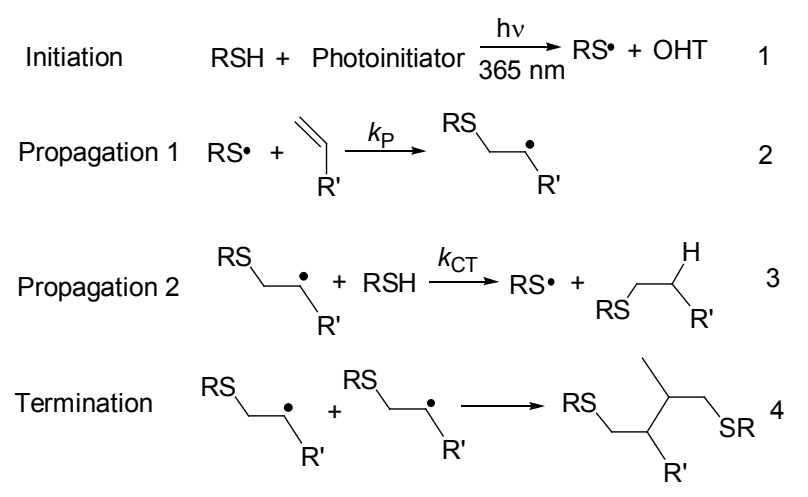

图 1 統基-烯光化学反应机理

Figure 1 Mechanism of thiol-ene photopolymerization

颈基-炔点击反应作为颈基-烯点击反应的补充在 新材料的合成方面表现出极大的优越性. 颈基和参键的 反应发现于 20 世纪 30 年代 ${ }^{[45,46]}$, 但是近几年 Lowe,

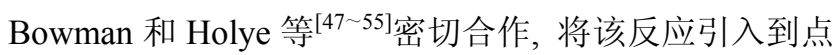
击化学领域. 与統基-烯点击反应相比, 颈基-炔点击反 应经历了颈基和参键的反应和颈基和双键的反应两个 过程, 反应过程如图 2 所示.

第一阶段在光化学或热催化作用下，硫醇化合物被 光引发剂分裂形成的自由基夺取一个氢原子, 形成硫醇 自由基，该自由基与烷基炔基团发生加成形成不稳定的 中间体硫醚-乙烯基自由基，硫醚-乙烯基自由基从附近 的硫醇化合物上夺取一个氢原子，形成颈基烯基醚中间 体. 第二阶段是一个統基-烯点击化学过程(其机理过程 如图 1 所示). 中间体颈基烯基醚进一步和巯基化合物 发生光化学反应的速率常数是第一步颈基-炔光化学反 应生成颈基烯丙基醚反应速率常数的三倍. 亦即颈基炔的光化学反应中第一步加成反应为慢反应或决速步. 而颈基-烯光化学过程和颈基-炔光化学过程相比, 颈基 -烯的光化学反应速率常数是巯基-炔光化学反应速率 常数的三倍, 而巯基-炔光化学反应过程与巯基-烯光化 学反应过程中发生点击反应数目相比, 其过程中一个参 键聚合单体可以和两个颈基化合物分子发生点击反应. 因此，颈基-炔点击反应在制备高交联密度的交联网络 聚合物 ${ }^{\left[{ }^{[2]}\right.}$ 和超支化结构聚合物中有较大优势，极大的 扩展了颈基点击反应在合成各向异性的新材料的应用 

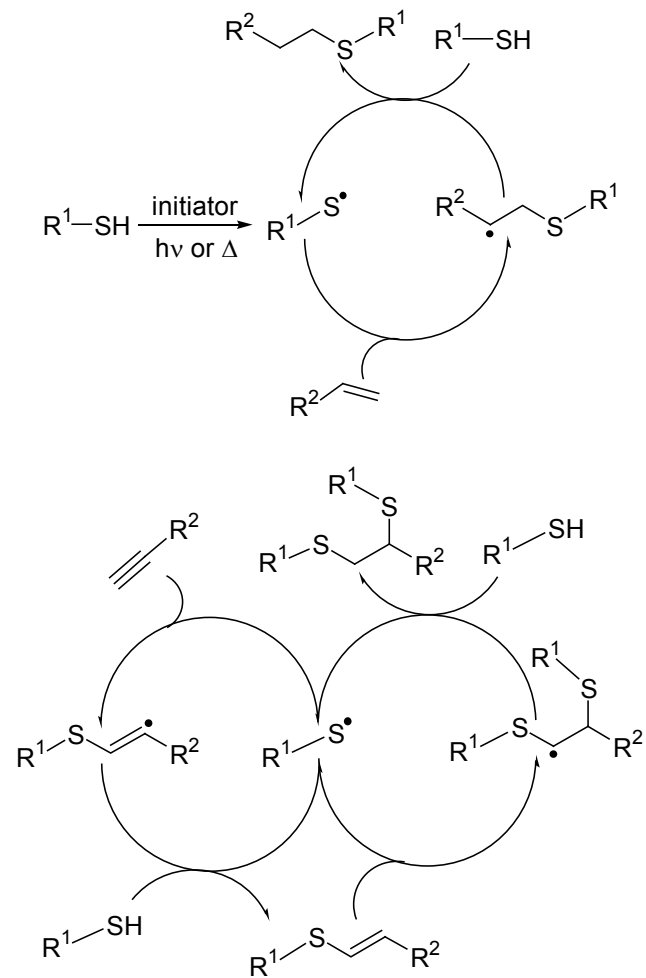

图 2 統基-烯点击化学(上)与巯基-炔点击化学(下)反应机理 特点

Figure 2 Traits of reaction of thiol-ene (up) and thiol-yne (down) click chemistry

范围.

鉴于上述两种类型颈基点击反应已经广泛的应用 于功能有机材料的设计和合成中, 且相关工作的总结较 少, 本文着重就上述两种点击反应在制备功能性聚合物 微球、两亲性的嵌段聚合物、分子器件材料、高度支化 聚合物等领域及化学修饰与改性方面进行了评述, 并总 结了近几年来颈基-烯/炔点击反应的在新材料的设计和 合成中的最新进展

\section{2 鋶基-烯/炔点击化学的应用}

\section{1 功能性微球的制备}

有机聚合物微球在色谱填料, 固相合成载体, 生物 医学，信息工程等科技领域得到越来越广泛的应用. 目 前聚合物微球的合成方法呈现出许多新兴的合成方法, 如模板聚合, 分子自组装, 微流体以及可控自由基活性 聚合. 相比较这些新兴的合成方法, 颈基点击化学以其 反应条件简单，反应快速(自由基反应机理)，过程可控 (光引发), 选择性好(巯基一烯或颈基-炔特异性反应), 产率高的优势在聚合物微球制备领域受到广泛关注.

Korthais 等 ${ }^{[63]}$ 在分散聚合体系中利用颈基-烯点击 化学制备了聚丁二烯纳米粒子, 将 1,2-聚丁二烯在溶剂 中形成直径为 $13 \mathrm{~nm}$ 的单体胶束溶液, 在溶液中加入含
有亲水性或憎水性基团的颈基化合物和水溶性自由基 引发剂 VA-057, 在 $50{ }^{\circ} \mathrm{C}$ 环境下反应 $20 \mathrm{~h}$, 形成了粒径 分布为 $16 \sim 104 \mathrm{~nm}$ 的功能化聚合物纳米粒子，而在不 加入颈基化合物的空白试验中所得纳米粒子的直径为 13 15 nm，与 1,2-聚丁二烯胶束的粒径相当，由此可以 证明由統基-烯点击化学反应成功得到了目标纳米粒子, 如图 3 所示.
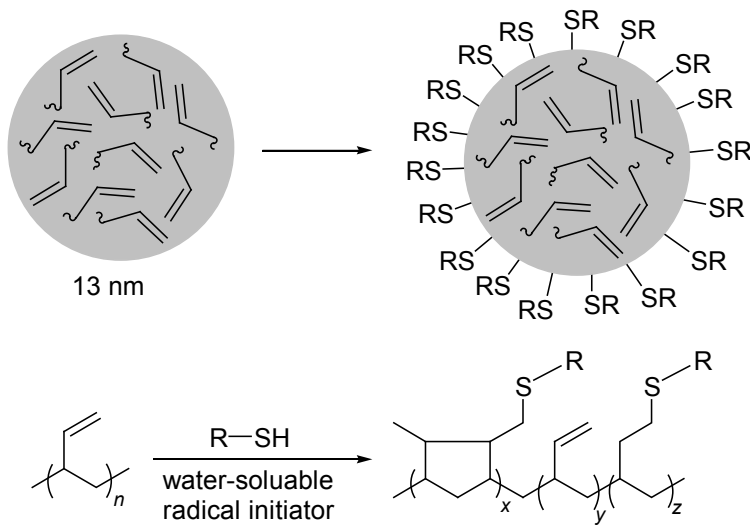

图 3 Thiol-ene 点击反应制备 1,2-聚丁二烯纳米粒子

Figure 3 Preparation of 1,2-polybutadiene nanoparticle via thiol-ene click chemistry

Prasath 等 ${ }^{[64]}$ 报道了微流体器件中利用颈基-烯/炔 点击化学制备无孔或多孔性的聚合物微球，将含有光引 发剂的四官能团統基化合物季戊四醇四(3-颈基丙酸 酯)(PETMP)、聚合单体和分散剂混合反应相逐滴注入到 微流体中的连续相中, 如图 4 所示, 紫外光辐射引发点 击反应，成功制备了粒径为 $210 \sim 600 \mu \mathrm{m}$ 的单分散的聚 合物微球，分析显示两种颈基点击反应制备的聚合物微 球球形度好，粒径均一，不同的是颈基一炔点击反应制 备的聚合物交联密度大; 用该方法制备的聚合物微球粒 径结构可控，表面官能团多样化，制备周期较短，在固 相合成，色谱分离，生物偶联和催化领域有广阔的应用 前景.

颈基-烯/炔点击反应除了能够成功制备无孔或多孔 性的聚合物微球外，颈基一烯/炔点击反应亦以其反应过 程的颈基与特定官能团反应的特异性和光引发自由基 反应的可控性即精确性，在制备尺寸精确和形状特定的 聚合物纳米微球具有独特的优势. Harth 课题组 ${ }^{[65,66]}$ 通过 点击化学对受控制的分子内交联制备不同大小的聚酯 纳米颗粒进行了研究, 首先用 $\delta$-戊内酯与 $\alpha$-烯丙基- $\delta$ 戊内酯在乙醇和四氢呋喃混合溶剂中, $105{ }^{\circ} \mathrm{C}$ 辛酸亚锡 催化反应 $48 \mathrm{~h}$ 形成线性共聚物聚 $(\delta$-戊内酯- $\alpha$ - 烯丙 基- $\delta$-戊内酯); 之后在线性共聚物溶液中加入 $2,2^{\prime}-(1,2-$ 乙二基双氧代)双乙硫醇(其反应过程如图 5A 所示). 实 验结果表明，通过颈基-烯点击反应使线性共聚物交联 


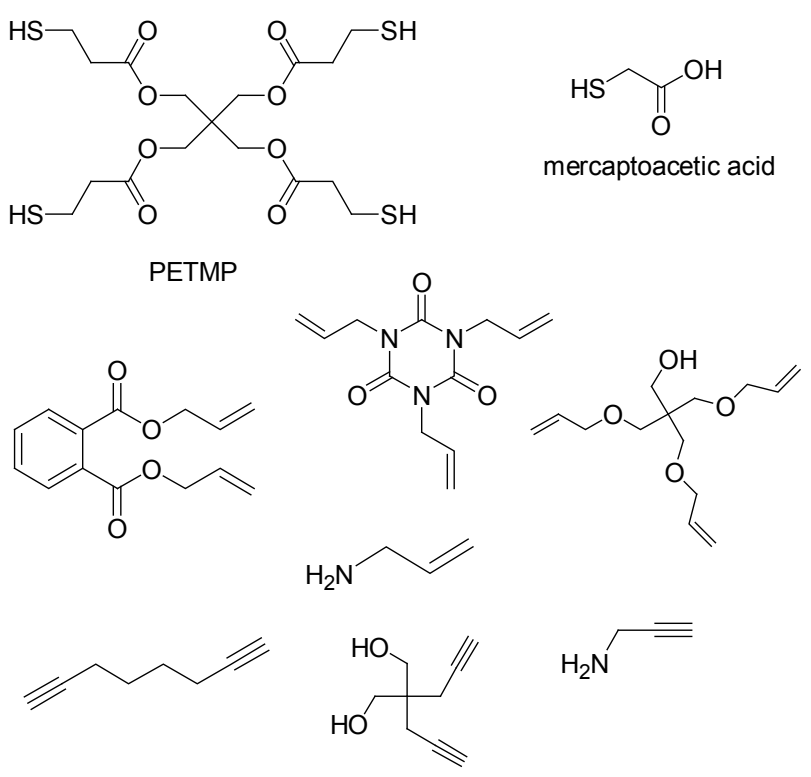

图 4 硫醇及聚合单体的结构及其缩写

Figure 4 Structure and acronym of thiol and monomers

形成纳米粒子, 动力学光散射, 透射电子显微镜研究表 明随着硫醇加入量从 1 增加到 $8\left(C_{\text {Thiol }} / C_{\text {Allyl }}\right)$, 形成的纳 米粒子的粒径从 $21 \mathrm{~nm}$ 增加到 $397 \mathrm{~nm}$, 而且粒径随反应 时间的延长增加有限.

\section{2 两亲性分子的合成及组装}

两亲性的嵌段聚合物具有在水或溶剂中发生分子 自组装，形成棒、球和泡囊等大分子分子聚集体的特性， 这种大分子聚集体在药物缓释、分离、分子催化等领域 有着广泛的应用前景. 而将点击化学应用于制备两亲性 的嵌段聚合物可以更加方便快捷地得到具有多功能性 的两亲性的嵌段聚合物, 目前有大量文献报道了点击化 学在制备两亲性的嵌段聚合物及其在大分子自组装中 的应用 ${ }^{[30,67 ~ 71]}$. 目前较多采用的是 $\mathrm{CuAAC}$ 点击反应制 备两亲性的嵌段聚合物, 然而铜催化 CuAAC 反应由于 $\mathrm{Cu}(\mathrm{I})$ 催化剂具有生物毒性，同时难以除去等缺陷以及 叠氮化学物的危险性等因素, 使其在应用方面有所限 制. 而巯基点击化学以其简便、低毒、高效的反应特点 可以更好地应用于制备两亲性的嵌段聚合物, 并且颈基 和双键或参键在热环境和光照情况下均能快速的发生 加成反应, 所以颈基点击化学成为近年来特定结构和功 能性聚合物制备的热点.

Chen 等 ${ }^{[72]}$ 以二硫代苯甲酸枯酯为 RAFT 聚合链转 移剂制备两嵌段的线性聚合物 Poly(DEGMA- $b$-HEMA), DMAP 作为催化剂, 4-戊烯酐处理嵌段共聚物引入双键 官能团, 在光照环境下加入葡硫糖单元得到葡萄糖修饰 的线性聚合物. 用颈基-烯点击反应制备的该线性嵌段 共聚物低临界共溶温度约为 $29{ }^{\circ} \mathrm{C}$, 当该嵌段共聚物溶

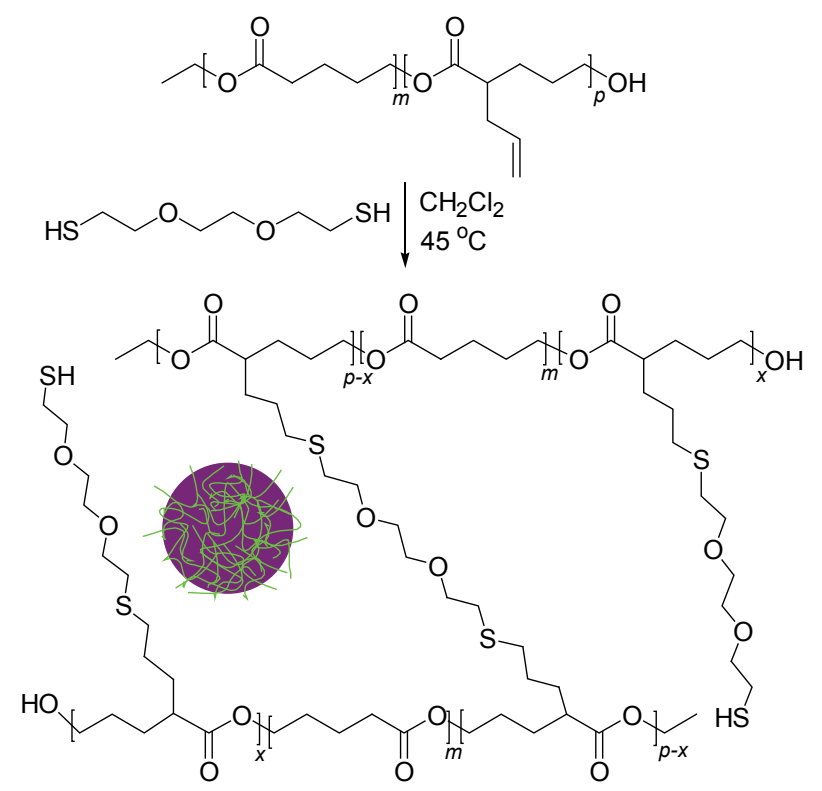

(A)颈基烯点击化学过程(Thiol-ene chemistry)

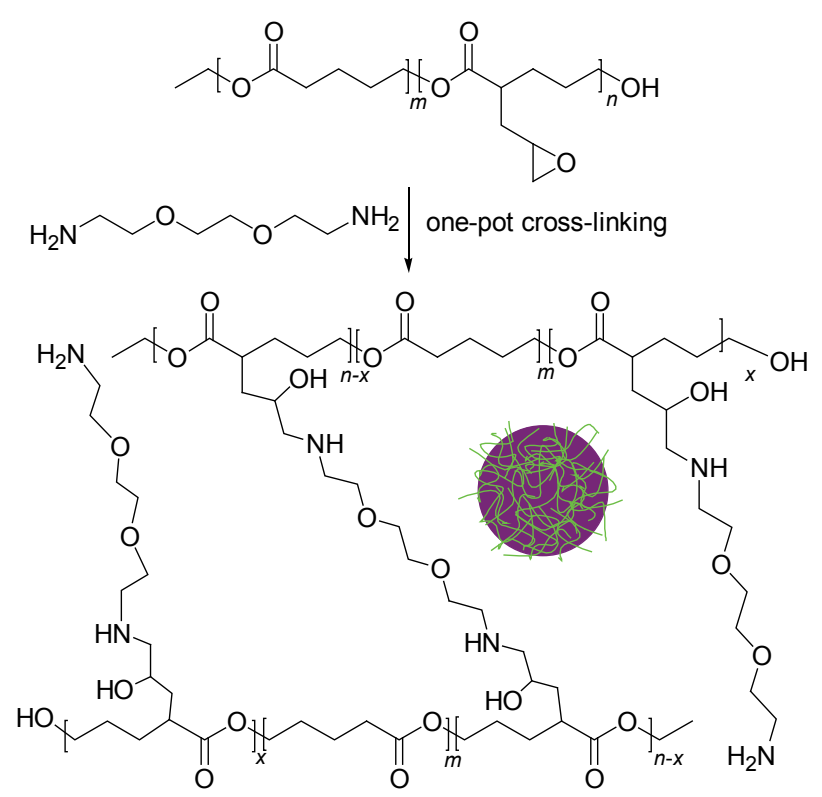

(B)环氧一胺交联微球(Epoxy-amina)

图 5 线性共聚物交联制备纳米材料

Figure 5 Preparation of nanoparticle via intermolecular cross-linking facilited with click chemistry

液温度在 $40{ }^{\circ} \mathrm{C}$ 以上时可以自组装形成聚合物纳米粒子, 动力学光散射仪(DLS)和 TEM 测试结果显示这种温敏 性聚合物纳米粒子的存在，说明了颈基-烯点击化学成 功的制备了两亲性的聚合物链.

Bulmus 课题组 ${ }^{[73]}$ 利用聚合单体吡啶二硫化甲基丙 烯酸乙酯(PDSM)作为颈基来源，在温和的还原条件下 $\mathrm{PDSM}$ 中 $\mathrm{S}-\mathrm{S}$ 键开裂形成颈基，这就为颈基-烯点击 反应提供了颈基基团. 首先在链转移的引发下 PDSM 聚 
合成线性聚合物, 在三(2-羰基乙基)磷盐酸盐(TCEP)弱 还原剂的存在下与丙烯酸化的 PEG 或 4-戊烯酸酯 PEG 链反应得到 PEG 修饰的聚合物链. 以该方法制备的 PEG 修饰的线性聚合物链在水溶液中能自组装成聚合 物纳米粒子, DLS 测得聚合物纳米粒子的平均水力学直 径为 $(99 \pm 8) \mathrm{nm}$, 分散系数 $(\mathrm{PDI})$ 为 $(0.22 \pm 0.02) \mathrm{nm}$. 此 外, 该课题组 ${ }^{[74]}$ 最近报道了采用颈基-烯点击反应制备 新型 PEG 和葡萄糖修饰的线性聚合物, 其反应过程如 图 6 所示. 用该方法制备得到的新型 PEG 和葡萄糖修饰 的线性聚合物链在水中能够自组装成纳米粒子, 平均水 力学直径为 $(192 \pm 28) \mathrm{nm}$.
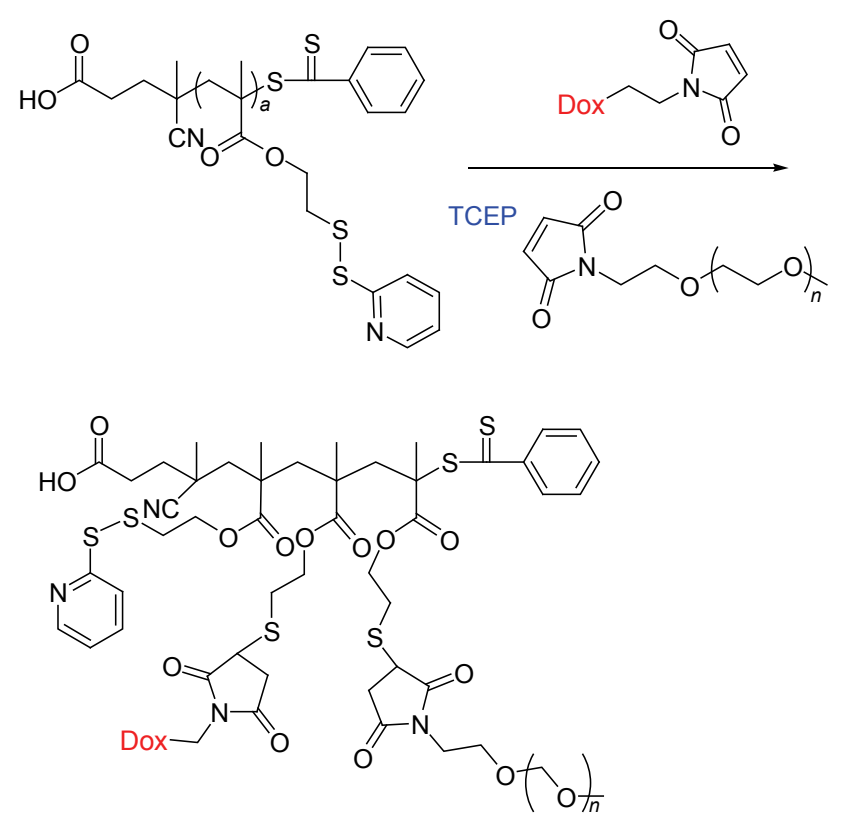

图 6 活性聚合鋶基烯点击化学制备负载抗生素纳米粒子

Figure 6 Scheme of nanoparticle burdened antibiotics via living polymerzation facilitated with click chemistry

颈基点击化学除了制备两亲性嵌段聚合物之外, 大 量文献报道了颈基点击化学与控制聚合的结合应用研 究.

控制聚合以其优异的反应特性在合成特定结构的 聚合物和分子量可控聚合物领域受到了广泛关注, 而将 点击化学结合控制聚合应用于制备具有多种物理化学 性质的功能性聚合物将呈现出更大优势. 目前, CuAAC 点击反应已经在该领域中开展了大量研究 ${ }^{[75 \sim 84]}$. 然而, $\mathrm{CuAAC}$ 点击反应使用了重金属 $\mathrm{Cu}$ 作为催化剂, 后处理 困难，限制了其在凝胶电解质等能源电子工业领域的应 用. 颈基点击化学热环境和光照情况下均能快速的发生 加成反应 ${ }^{[85 ~ 87]}$, 并不使用重金属类催化剂, 正是这一优 点使得颈基点击化学结合控制聚合成为今年来功能性 聚合物制备的热点, 尤其是在药物缓释载体材料制备领 域 ${ }^{[88 \sim 90]}$.
目前, 研究较多的是 RAFT 控制聚合 ${ }^{[1 ~}$ 93] 、ATRP 控制聚合 ${ }^{[94,95]}$ 与颈基点击化学结合研究. 其中, RAFT 活性聚合链转移剂结构中的二硫代酯基在 $\mathrm{NaBH}_{4}$, TCEP 等弱还原剂的作用可有效的转化为颈基, 使得颈 基点击化学与 RAFT 控制聚合相结合成为可能，如图 7A 所示. ATRP 结合颈基点击化学研究方面, Yagci 等 ${ }^{[96]}$ 首次报道了2-澳乙酸乙酯引发剂引发ATRP控制聚合形 成的大分子中间体，可经过硫腿等反应将卤素转变为端 颈基的大分子(反应过程如图 7B 所示), 使 ATRP 控制聚 合与颈基点击化学结合成为了可能.

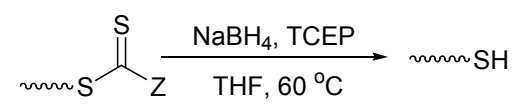

A

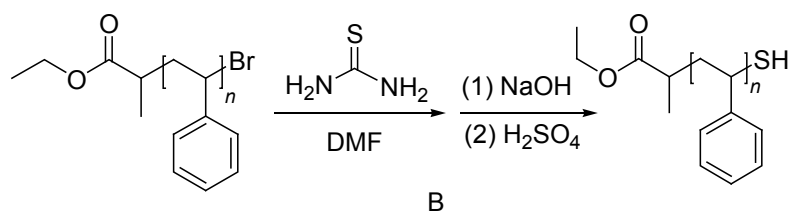

图 7 活性聚合中间体转化为端放基化合物示意图

Figure 7 A schematic of linear polymer transformed from living polymerzation intermediate

表 1 列出了目前报道的几种控制聚合结合統基点击 化学在不同结构形态聚合物的合成和材料修饰中的应 用，可以看出主要集中在颈基点击化学结合 RAFT 控制 聚合的研究.

除这两种典型控制聚合外，催化链转移活性聚合 (catalytic chain transfer polymerization)与颈基点击化学 结合制备功能性线性聚合物是最近报道的一种新方法. 该方法和以上两种控制聚合结合过程不同, ATRP 和 RAFT 控制聚合合成的线性聚合物的端基先转化为颈 基，再利用颈基-烯点击反应与双键分子片段结合完成 分子体系的构建; 而通过催化链转移活性聚合(CCTP) 控制聚合制备的线性聚合物链端中含有未反应的双键, 可以和多种硫醇化合物利用颈基点击反应制备有机聚 合物材料.

Nurmi 等 ${ }^{[97]}$ 以报道了催化 CCTP 和颈基-烯点击化 学制备了功能聚合物链. 其制备过程是首先以甲基丙烯 酸类单体 1 在钴 II 肜氟化嗍配合物 (CoBF) 催化剂、 $2,2^{\prime}-$ 偶氮二异丁腈 $(\mathrm{AIBN})$ 为引发剂, 由催化链转移聚合法 合成了线性聚合物链，该线性聚合物链分子量分布均 匀, 链中保存双键官能团, 第二步将小分子 $(\mathrm{R}-\mathrm{SH})$ 通 过颈基一烯点击反应引入到线性聚合物链中，成功的制 备了硫醇化合物修饰的结构精确的聚合物材料(图 8).

Soeriyadi 等 ${ }^{[98]}$ 以温敏性的大分子单体在 $\mathrm{CoBF}$ 催化 剂和 AIBN 引发剂作用下，通过 CCTP 控制聚合合成了 侧链为亲水性 PEG 的梳形聚合物链, 该线性聚合物链 


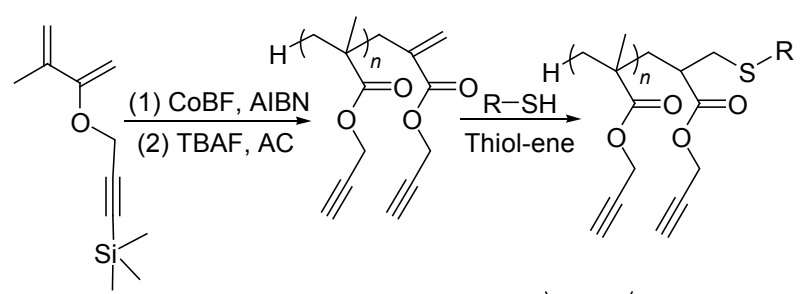

$\mathrm{R}-\mathrm{SH}: \mathrm{HS} \longrightarrow$

CoBF :<smiles>OCCS</smiles><smiles>CC1=C(C)N2O[PH](F)(F)ON3C(C)=C(C)[N+]4(CN1OB(F)O4)C23O</smiles>

图 8 端基官能团聚合物的合成

Figure 8 Synthetic approaches to end-functionalized glycopolymers

的最低临界溶解温度(LCST)分布于 30 至 $90{ }^{\circ} \mathrm{C}$, 而聚合 物链中保存的双键与多种硫醇化合物发生颈基一烯点击 反应得到了小分子修饰的温敏性聚合物(图 9), 结果显 示疏水性硫醇的修饰可以降低线性聚合物的 LCST, 而 亲水性硫醇的修饰有相反的作用.

以上表明, CCTP 活性聚合和颈基-烯点击化学的结 合能成功制备结构和功能各异的聚合物材料, 具有较大 的应用前景.
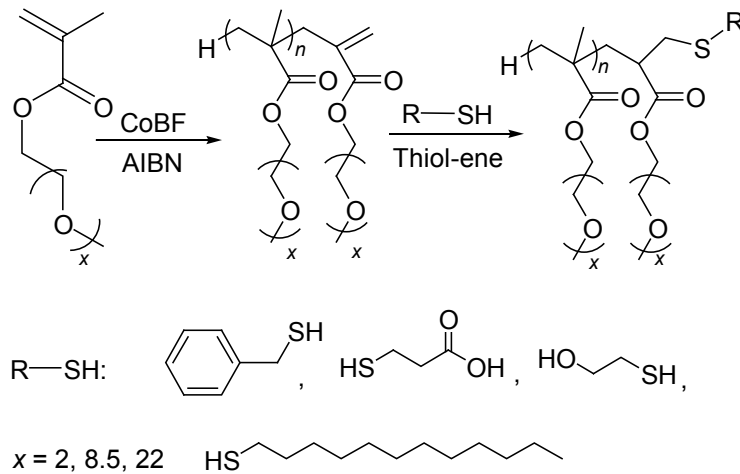

图 9 温敏型聚甲基丙烯酸寡聚乙二醇酯线性聚合物的修饰

Figure 9 Synthesis and modification of linear thermoresponsive poly(oligo(ethylene glycol) methacrylate)

\section{3 分子器件材料的制备}

超分子聚轮烷和索烃类有机材料是一类构建分 子器件优异材料, 其具有信息传递更加方便快捷的优 势, 然而因其具有复杂的结构及其特定的功能, 一直是 在材料制备领域的一大难题. 传统的合成方法是将不完 整的环状受体分子与哑铃状分子在疏水空穴效应、静电 或空穴结构的选择性作用的驱动下组装成不完整的轮 烷或索烃体系，再将环状受体分子闭合形成完整的超分

表 1 控制聚合和巯基-烯/炔点击化学

Table 1 Specimens of controlledpolymerization facilitated with thiol-ene/yne click chemistry

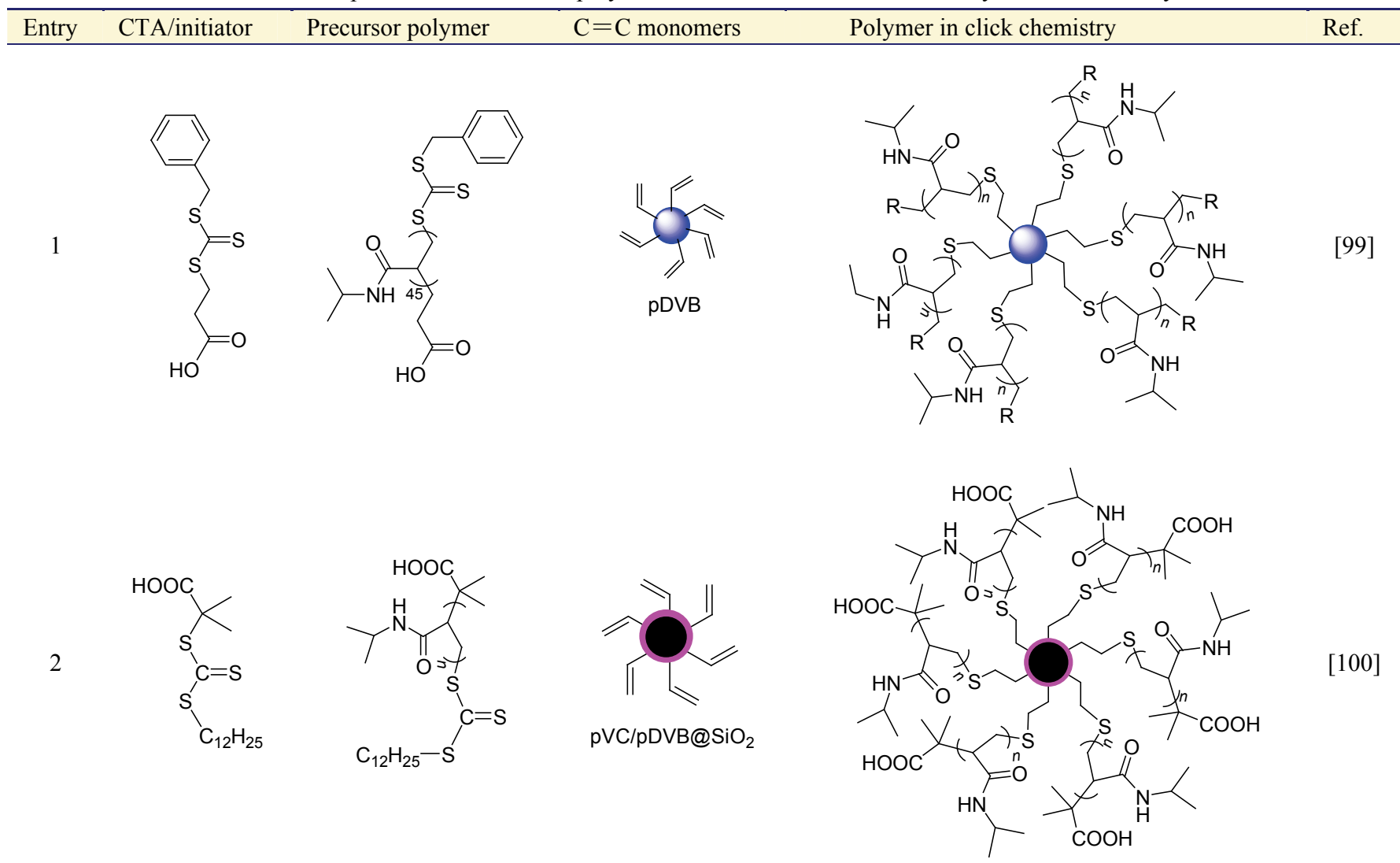




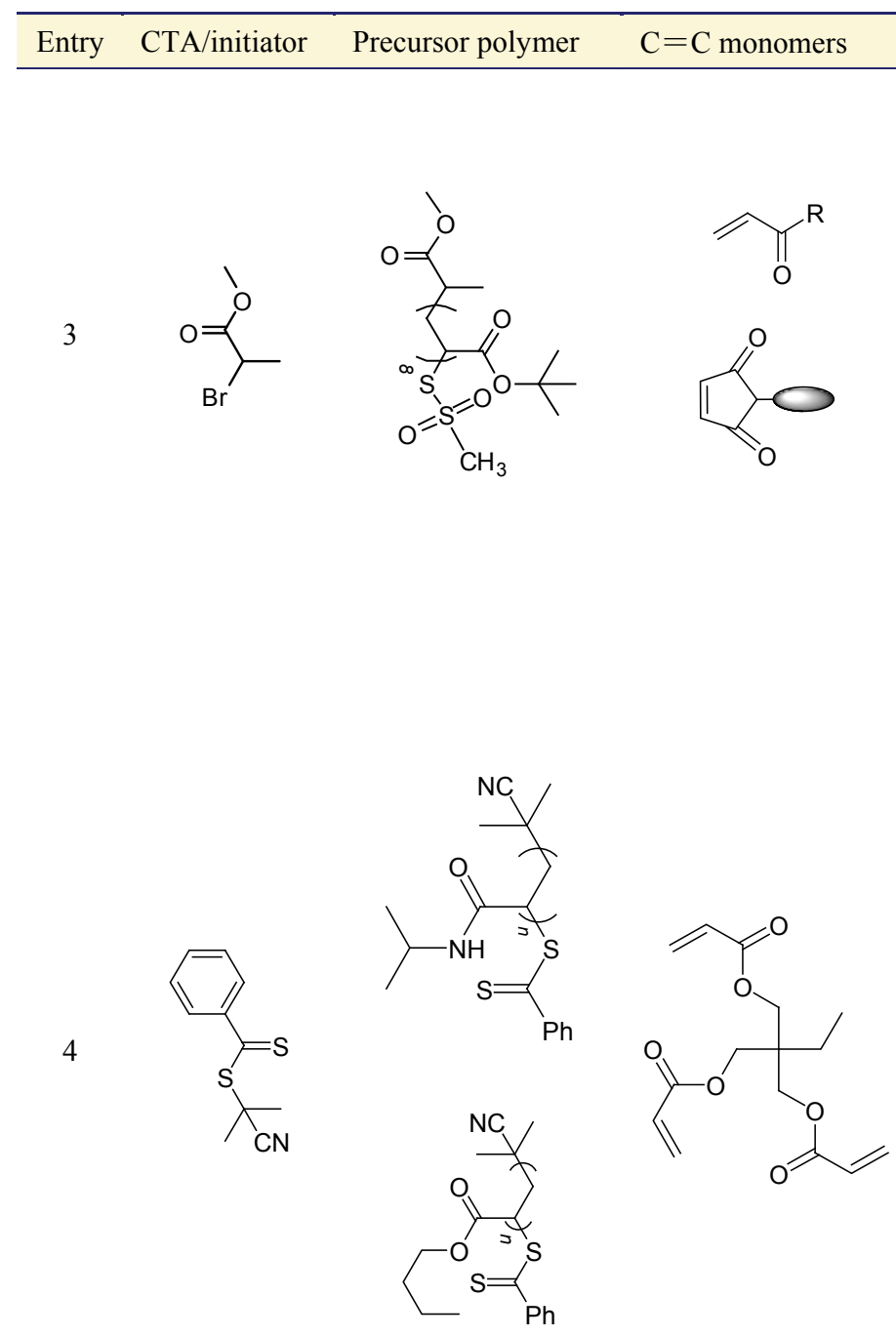

Polymer in click chemistry Ref.

续表
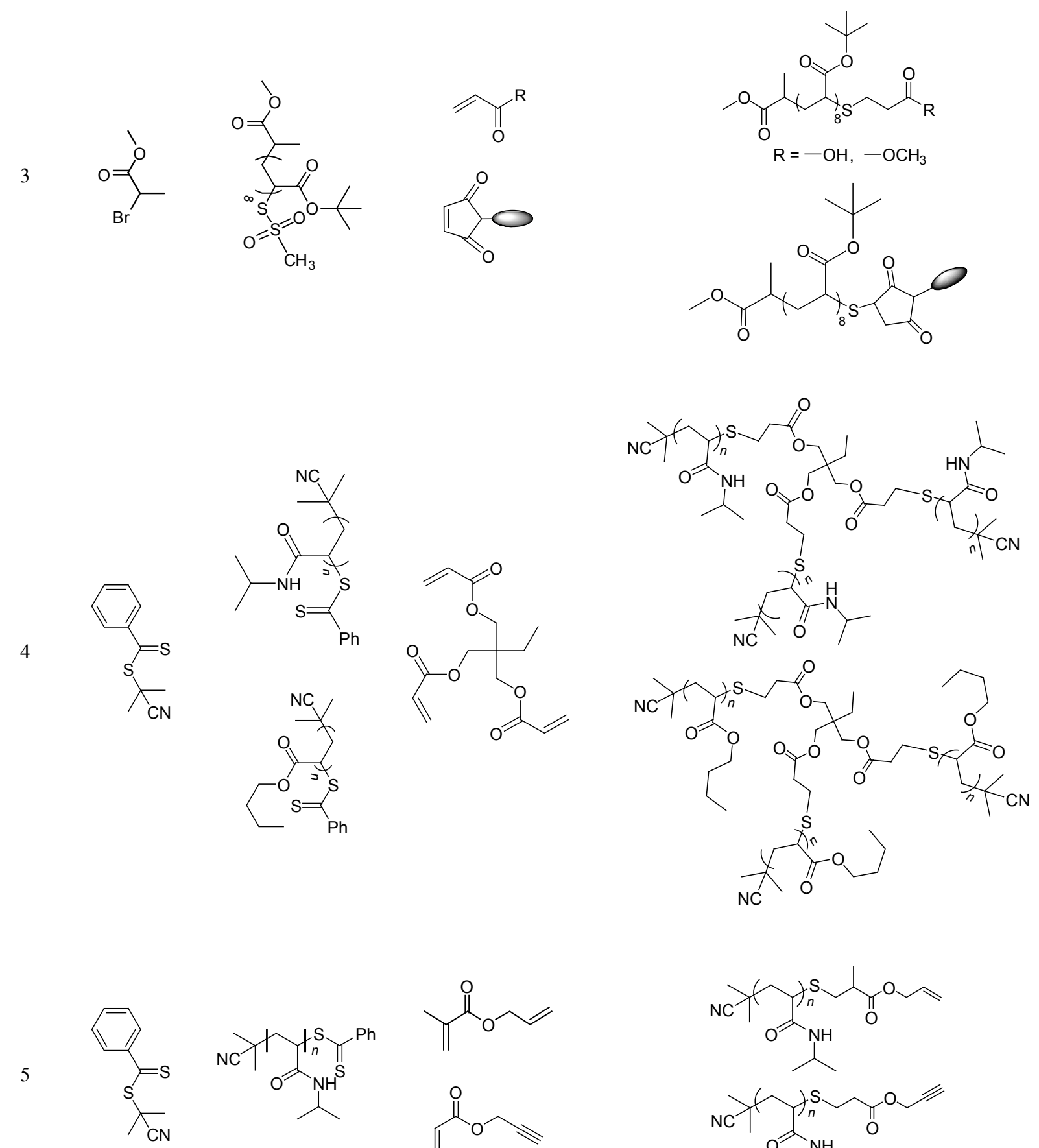<smiles>CC(C)NC(=O)C(CC(C)(C)C#N)SC(=S)c1ccccc1</smiles><smiles>C#CCOC(=O)C=C</smiles><smiles>C#CCOC(=O)CCSC(CC(C)(C)C)C(=O)NC(C)C</smiles>

6<smiles>CCCCCCCCCCCCCCC(C)(C)C(=O)O</smiles><smiles>CC(C)NC(=O)[C@H](S)CC(C)(C)C(=O)O</smiles><smiles>O=C1C=CC(=O)N1CCOCCN1C(=O)C=CC1=O</smiles><smiles>CC(C)NC(=O)C(C)SC1CC(=O)N(CCOCCN2C(=O)C=CC2=O)C1=O</smiles> 


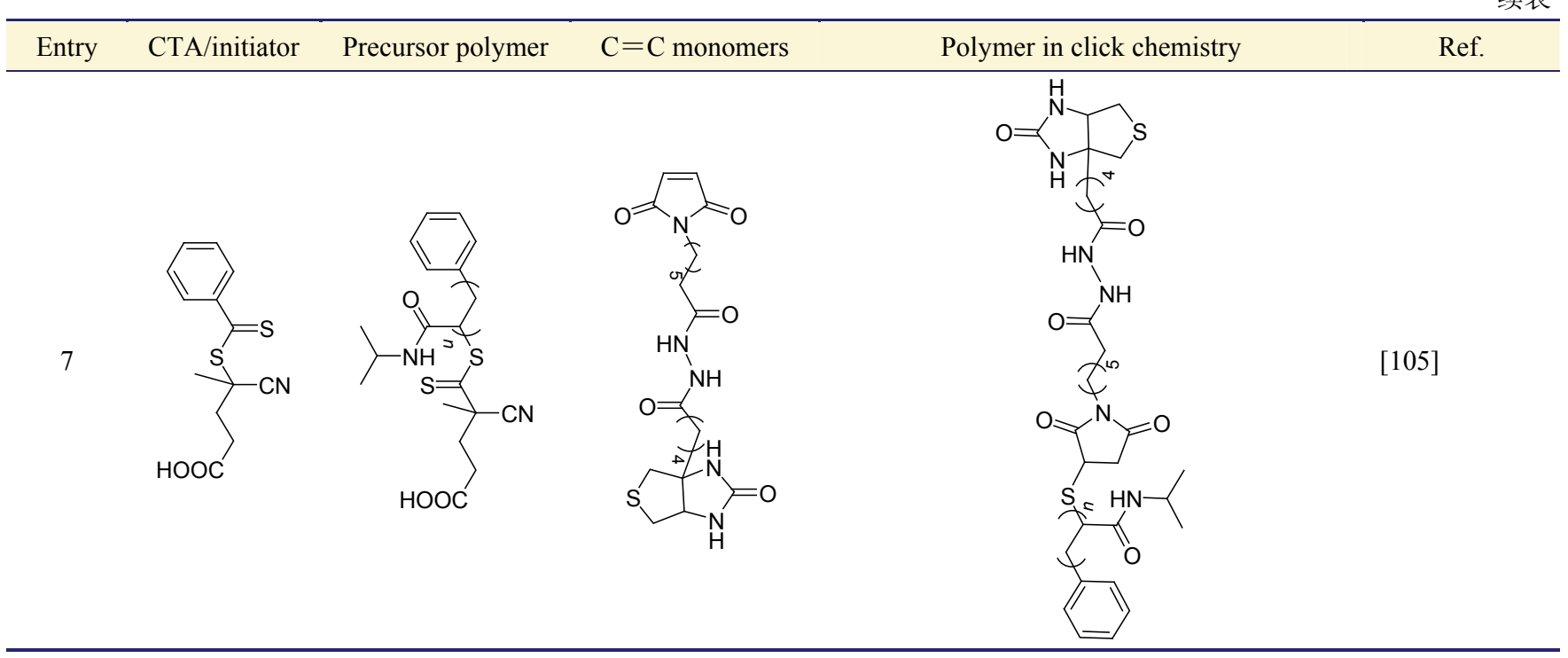

子体系, 这种合成方法产率低. 由于統基点击化学在化 学合成中具有的方便快捷和高效率等优点, 在制备超分 子聚轮烷和索烃类等分子器件材料中表现出明显地优 势. 这种新型的合成方法是将闭合的受体分子和含有官 能团的哑铃状分子某一片段组装成准轮烷超分子系统, 再利用颈基点击化学反应形成哑铃状或环状分子.

Liu 等 ${ }^{[106]}$ 采用准轮烷加塞方法成功制备超支化聚 轮烷. 该制备方法主要分为二个阶段, 第一阶段主要合 成了新型的含颈基 $\mathrm{AB}_{2}$ 型线性聚合单体 PA-PCL-SH, 其结构如图 10 所示; 第二阶段是该单体通过与 $\alpha$-环糊 精加成形成了聚轮烷的聚合单体 $\alpha$-CD-PA-PCL-SH (图 $10 \mathrm{C}$ ), 在安息香二甲醚(DMPA)光引发剂的作用下紫外 光引发聚合成聚轮烷的超支化聚合物 HPPRs, 其结构如 图 10E 所示. 实验结果表明, 该方法制备得到的超支化 聚合物 HPPRs 具有较高的产率.

Zheng 等 ${ }^{[107]}$ 采用颈基-烯点击反应成功合成了哑铃 状分子和两种新型冠醚衍生物的大环受体分子(图 11 所 示). 利用冠醚空穴结构选择性的直接组装成[2]-轮烷超 分子系统 R-1, R-2 和 R-3, 其反应过程如图 12 所示. 实 验结果显示 R-1 的产率达 75\%, 而 R-2, R-3 的产率各为 $30 \%$ 和 38\%, 这是由于二硫键取代冠醚衍生物大环受体 后, 二硫冠醚空穴受体与二芐铵盐阳离子之间的选择性 下降所致.

Zhou 等 ${ }^{[108]}$ 以颈基-炔点击反应闭合受体冠醚衍生 物大环的方法合成了 [2]-轮烷超分子系统 R-1, 其反应 过程如图 13 所示. 与传统的闭环方法相比, 该方法所得 的轮烷产率高达 $75 \%$, 反应时间也大大缩短至 $5 \mathrm{~min}$.

\section{4 点击化学与化学修饰及改性}

材料的应用范围取决于表面化学、物理性质和反应
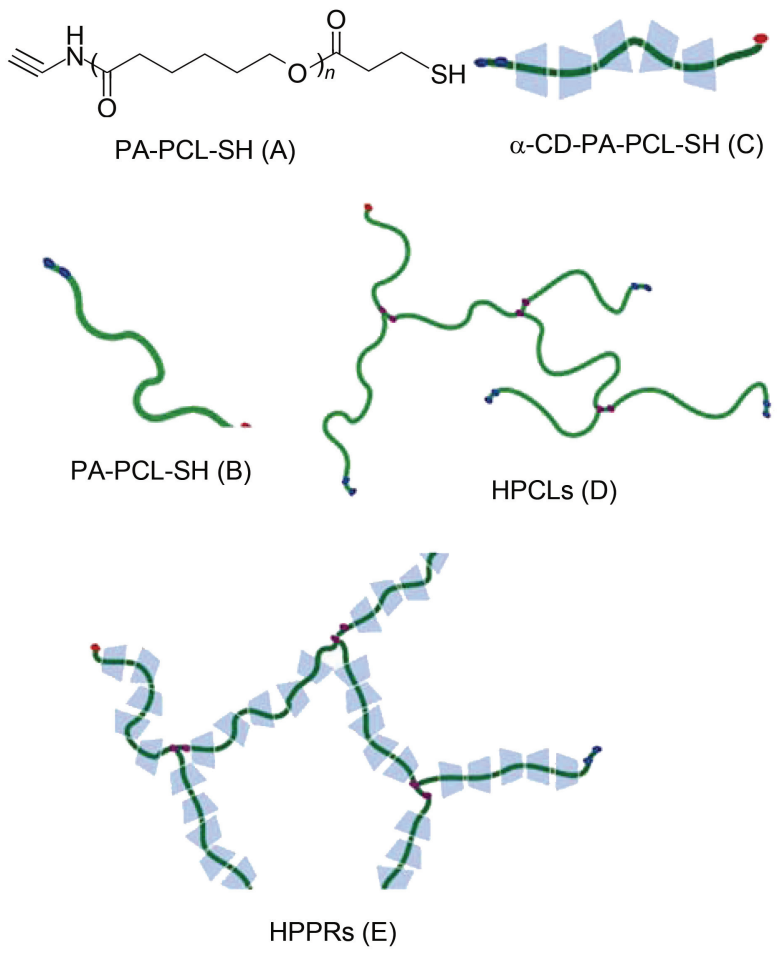

图 10 超支化聚轮烷大分子的合成

Figure 10 Synthesis of hyperbranched polypseudorotaxanes via thiol-yne click chemistry

性等多种因素, 对材料进行表面修饰不仅可以提供基材 表面官能团，而且能增强材料的附着力、亲水疏水性、 生物相容性、抗污性、表面硬度和粗粘度等性能. 通过 表面修饰可以使材料获得具有可设计性和反应性，而不 需要改变基材本身的性质. 近年来, 点击化学以其反应 条件简单，立体选择性强，符合原子经济等优点在表面 修饰改性方面具有很大的发展潜力. 其中, 統基-烯/炔 点击化学相比于其他类型的点击反应具有使用少量催 

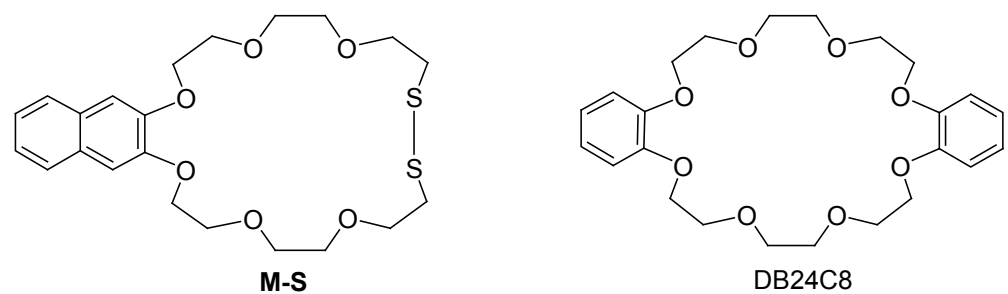

图 11 冠醚轮烷大环分子

Figure 11 Crown ether rotaxane macrocycle molecules<smiles>O=C(CS)OCCCOc1ccc(CNCc2ccc(OCCCOC(=O)CS)cc2)cc1</smiles><smiles>C=CCOc1ccc(C(c2ccc(C(C)(C)C)cc2)(c2ccc(C(C)(C)C)cc2)c2ccc(C(C)(C)C)cc2)cc1</smiles>

$$
\begin{aligned}
& \text { DMPA/DCM } \\
& \underset{\text { DB24CB or M-S }}{\stackrel{h v}{\longrightarrow}}
\end{aligned}
$$

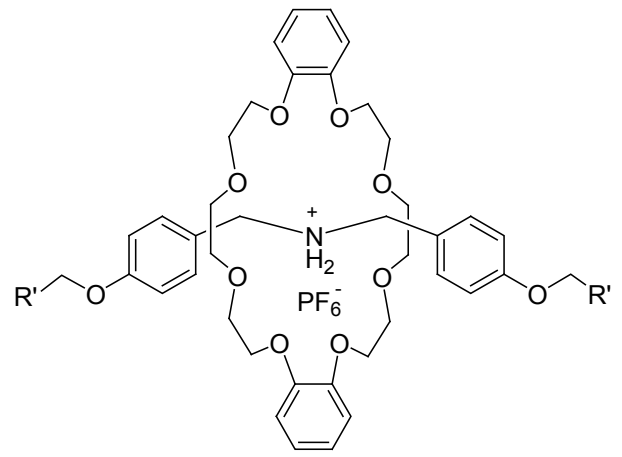

R-1<smiles>[R]COc1ccc(CCOCCOCCOc2cc3ccccc3cc2OCCOCCOc2ccc(OC[R])cc2)cc1</smiles>

R-2<smiles>[R]=CCC(=O)CSCCCOc1ccccc1</smiles><smiles>CC(C)(C)c1ccccc1</smiles><smiles>Oc1cc2ccccc2cc1OCCOCCOCCSSCCOCCOCCOc1cc2ccccc2cc1O</smiles>

M-S

图 12 轮烷的合成与颈基一烯点击化学

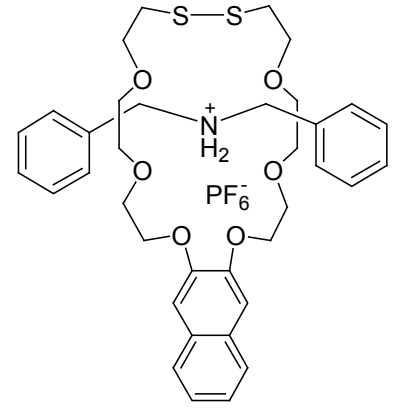

R-3

Figure 12 Synthesis of rotaxane via thiol-ene click 

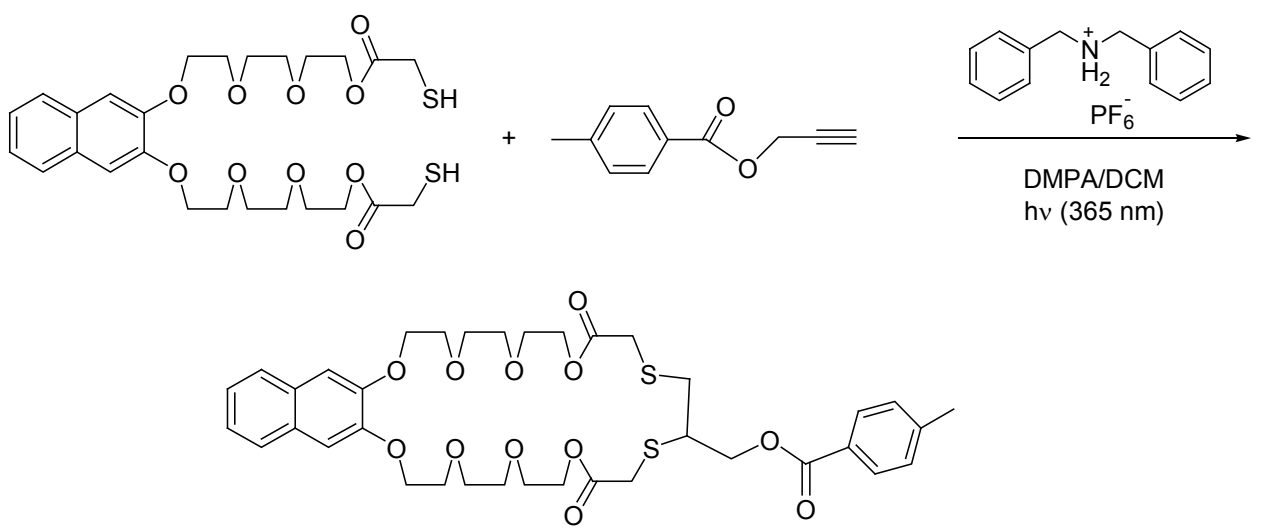

A
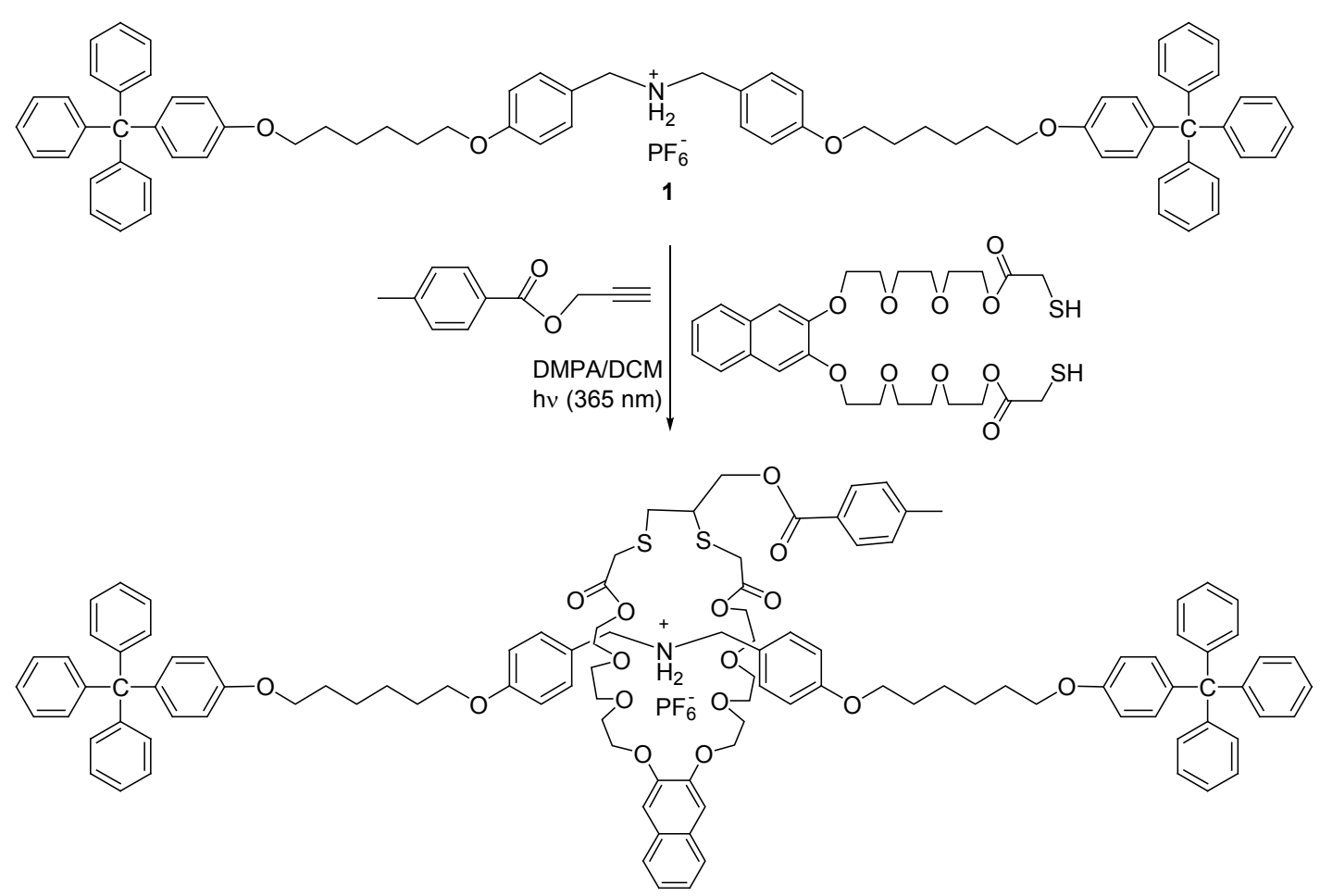

图 13 轮烷分子系统的合成与巯基-炔点击化学

Figure 13 Synthesis of rotaxane molecular system via thiol-yne click chemistry

化剂或不用催化剂等优点, 因此具有更好的生物相容 性, 成为实现基材的表面修饰改性的一种必然趋势.

一般巯基-烯点击化学表面修饰改性有聚合物偶联 反应修饰、表面单体接枝反应修饰和巯基点击反应交替 修饰三种主要方式, 其机理如图 $14^{[85]}$ 所示.

聚合物偶联反应修饰方面, 加州大学 Hawker 课题 组 ${ }^{[109]}$ 在该领域进行了较为深入研究, 最近报道了一系 列采用颈基-烯点击反应修饰线性聚合物的研究. 修饰 线性聚合物侧链: 首先采用活性聚合和开环聚合得到含 有侧链端位双键的线性聚合物, 然后在光引发剂 DMPA 和紫外光辐射作用下分别用 5 种颈基化合物 7 11(如图 15 所示)通过統基-烯点击反应对其侧链进行功能化修
饰，得到经修饰后的聚合物 $1 \sim 3$. 修饰线性聚合物主 链: 首先合成了三种含有端位双键的线性聚合物, 采用 同样的点击反应修饰方法得到了不同官能团的大分子 $4 \sim 6$.

上述两种线性聚合物修饰的转化率均在 $95 \%$ 以上, 表现出良好的修饰效果.

Heggli 等 ${ }^{[10]}$ 报道了在聚苯乙烯微球的表面利用統 基-烯点击化学反应制备了表面富含生物活性基团的聚 苯乙烯微球, 并且以活化的聚苯乙烯微球与氨基酸进行 偶联, 并研究了其在生物医学领域中的应用. Rutledge 等 ${ }^{[111}$ 通过巯基-烯点击化学反应修饰改性具有超顺磁 性的 $\mathrm{Fe}_{3} \mathrm{O}_{4}$ 纳米粒子, 其反应过程如图 16 所示. 经修饰 


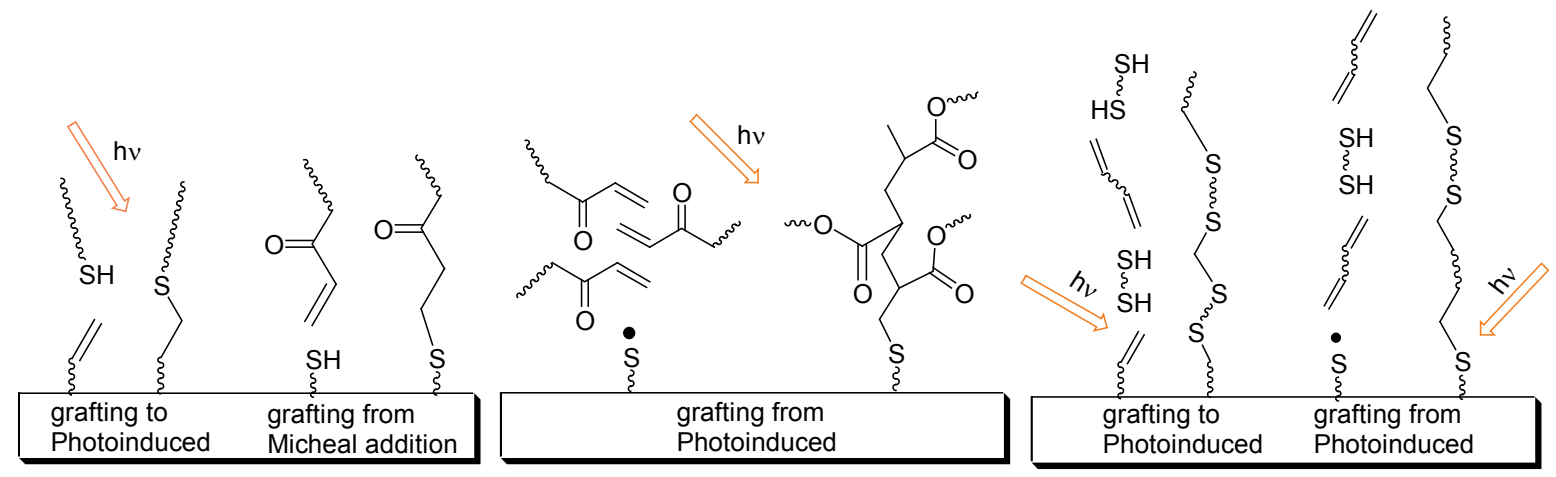

图 14 統基-烯点击化学表面修饰方式

Figure 14 Methods of surface modification via thiol-ene click chemistry<smiles>[R]CCCCOCc1ccc(C(C)C)cc1</smiles><smiles>[R]CCCCOC(=O)C(C)CC(C)(C)C</smiles>

2<smiles>[R]CCCCC(NC(C)=O)OC(=O)COC</smiles>

3<smiles>[R5]CCCOC(=O)C(C)(C)CC(C)(C)C(Br)c1ccccc1</smiles><smiles>[R5]CCCCOC(=O)C(C)(C)C(C(=O)OC)C(Br)C(=O)OC</smiles><smiles>[R5]CCCOCCOC</smiles><smiles>O=C(O)CS</smiles><smiles>CCO[Si](OCC)(OCC)OCCCS</smiles><smiles>SC12CC3CC(CC(C3)C1)C2</smiles>

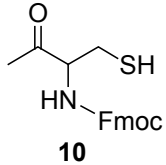<smiles>OCC(O)CS</smiles>

图 15 統基-烯点击化学修饰合成功能性线性聚合物

Figure 15 Synthesis of functional linear polymer via thiol-ene click chemistry

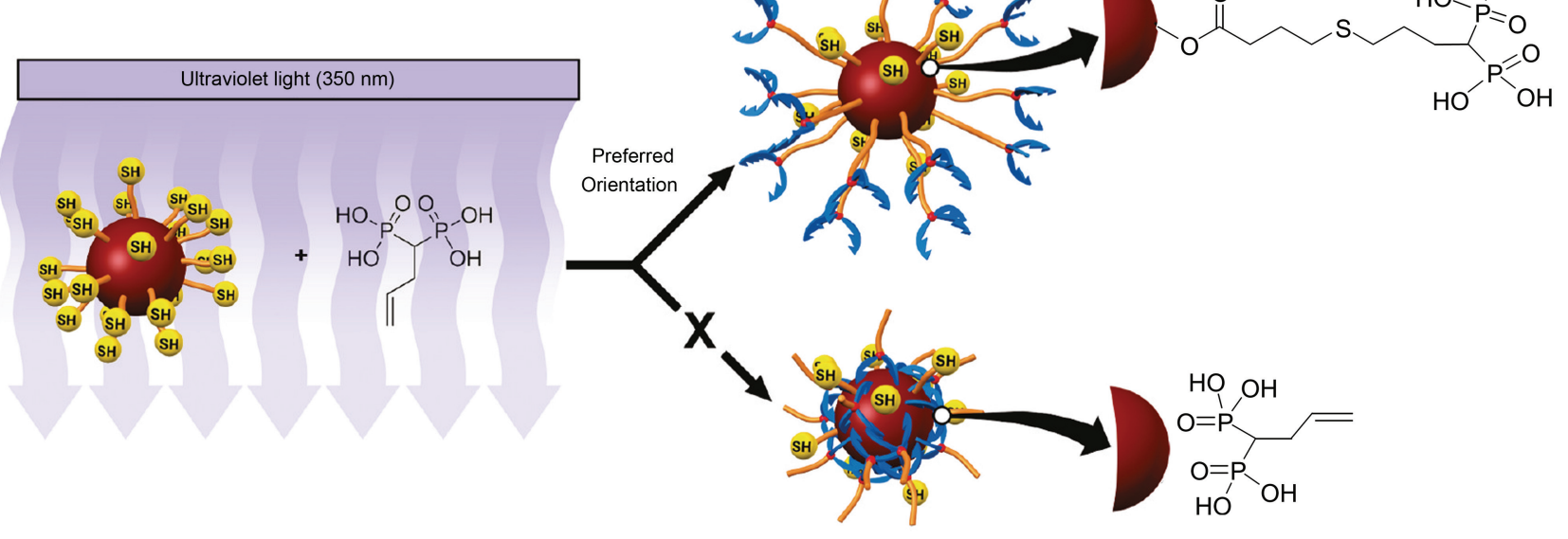

图 16 統基-烯点击化学修饰 $\mathrm{Fe}_{3} \mathrm{O}_{4}$ 纳米粒子 [111]

Figure 16 Modification of $\mathrm{Fe}_{3} \mathrm{O}_{4}$ nanoparticle via thiol-ene click chemistry 
后的 $\mathrm{Fe}_{3} \mathrm{O}_{4}$ 纳米粒子表面富含二膦酸基团, 从而能有效 吸附 $\mathrm{La}, \mathrm{Eu}$ 等稀土元素和重金属元素, 在冶金领域和工 业废水处理方面有着广泛的应用前景.

Berkel 等 ${ }^{[112]}$ 在乳液聚合体系中合成了聚二乙烯基 苯包裹 $\mathrm{Au}$ 纳米粒子的微球, 微球表面含有较多的未反 应的双键, 颈基化的 PEG 链与微球表面的双键发生颈 基-烯点击化学反应, 改性后微球在水中有很好的分散 性. Salinas 等 ${ }^{[13]}$ 将颈基化的氨基酸序列与丙烯酸化的 PEG 链通过統基-烯点击化学反应形成线性共聚物, 这 种聚合方法为 PEG 凝胶体系中高效的共价偶联细胞特 异性氨基酸提供了简捷的方法.

表面单体接枝反应修饰方面，此类型反应在制备核 壳结构的粒子方面应用较多. 但是受限于传统自由基聚 合过程中存在氧阻聚效应等缺点, 紫外光引发时接枝反 应速率较小, 因此目前颈基点击化学结合活性聚合在单 体接支反应修饰领域研究较为广泛.

颈基点击反应交替修饰方面, $\mathrm{Li}$ 等 ${ }^{[114]}$ 利用分子层 沉积法, 通过颈基-烯点击化学在硅基半导体材料表面 组装成聚合物薄膜. 首先, 通过化学作用在单晶硅板表 面形成 $\mathrm{Si}-\mathrm{H}$ 键, 然后将二官能团烯烃的端位双键分别 与 $\mathrm{Si}-\mathrm{H}$ 键和二颈基化合物进行硅氢加成反应和颈基烯点击化学反应; 二官能团烯烃和二官能团硫醇再进行 交替反应最终在硅基表面发生分子层层自组装形成薄 膜. 此外, 还考察了不同烯烃和颈基化合物对自组装薄 膜形态和物理机械性能的影响. Bowman 课题组 ${ }^{[115,116]}$ 报 道了在双键修饰的 $\mathrm{SiO}_{2}$ 纳米粒子表面利用光催化的颈 基-烯点击反应线性聚合物薄膜. 第一步用巯基硅烷偶 联剂处理 $\mathrm{SiO}_{2}$ 纳米粒子, 使其表面颈基化, 第二步是二 官能团的颈基化物和二官能团的烯类聚合单体与光敏 剂在紫外光引发作用下在纳米粒子表面形成线性聚合 物薄膜, 利用酸性催化剂使使纳米粒子表面的线性聚合 物从纳米粒子表面裂解, 并测得线性聚合物的分子量分 布为 $M_{\mathrm{n}}=900 \sim 12600$ 和 $M_{\mathrm{w}}=1500 \sim 25700$, 并讨论了 颈基化合物和烯的摩尔比值对线性聚合物分子量的影 响.

\section{5 点击化学与高度支化聚合物}

\subsection{1 树枝状聚合物的制备}

Hawker 等 ${ }^{[117,118]}$ 最先报道将颈基烯/炔点击化学应 用到树枝状聚合物的制备中. 该课题组研究了統基-烯 和酯化反应来制备树枝状聚合物, 在以 $1,3,5$-三聚氧酸 三烯丙基酯 $3 \mathrm{~T}$ 作为聚合物的核方面进行了深入的研究. 其制备过程分为两种策略: 一种策略是以 $3 \mathrm{~T}$ 为核, 3-颈 基-1,2-丙二醇 (1-thioglycerol)和 4-戊烯䣶 (4-pentenoic anhydride)作为反应物, 通过两步交替颈基-烯点击化学 和酯化反应得到[G4]-(OH) 48 多羟基树枝状聚合物, 产
率高达以上 $90 \%$, 其反应过程如图 17 所示; 另一种策略 是采用新型的含有颈基的多羟基的 $\mathrm{AB}_{2}$ 单体代替 3-颈 基-1,2-丙二醇和 $\mathrm{CD}_{2}$ 双烯丙基醚酸酛单体来替代 4-戊烯

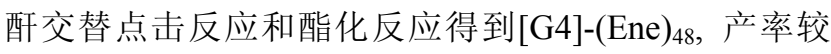
传统的方法制备的树枝状聚合物产率有较大提高.

$\operatorname{Rim}$ 等 $^{[119]}$ 成功合成了以 $1,3,5$-三丙烯酰基六氢$1,3,5$-三嗪为核的三臂星形低聚物. 其反应过程是第一 步通过颈基-烯点击化学将 2-颈基乙醇和核双键加成得 到羟基化合物，第二步是以丙烯酰氯酯化端差基，使其 转变为双键. 实验结果表明, 2-颈基乙醇和丙烯酰氯交 替官能化后测得 $[4 \mathrm{G}]-(\mathrm{OH})_{3}$ 低聚物分子量约为 1672 , 支 化结构较完整, 且各步反应具有较高的产率.

Son 等 ${ }^{[120,121]}$ 采用颈基-烯点击化学成功制备了含 硅树枝状大分子. 实验过程是以四氯硅烷为核, Grignard 反应和颈基-烯点击反应交替进行得到含硅的多官 能双键的树枝状聚合物, 其反应过程如图 18 所示, 得到 的第五代聚合物分子量为 89227.98 , 产率达 $87 \%$, 结果 表明该方法可以得到支化度完整，产率高的树枝状大分 子.

颈基-烯和酯化反应结合能高效的制备树枝状聚合 物, 但是酯化反应时间周期较长, 产物提纯不易. 颈基烯点击化学和 CuAAC 点击反应相结合制备树枝状聚合 物缩短了制备周期, 并且产率较颈基-烯和酯化反应所 得的枝化结构大分子高. Hult 课题组 ${ }^{[122]}$ 报道了在一天 之内合成 6 代的树枝状聚合物, 以 $3 \mathrm{~T}$ 为聚合物核, 采用 新型含有颈基的二颈基 $\mathrm{AB}_{2}$ 单体 12 和含有炔基的二烯 $\mathrm{CD}_{2}$ 单体 13, 颈基-烯和 $\mathrm{CuAAC}$ 点击反应交替进行得到 树枝状聚合 14(图 19). 实验结果显示 TOF 检测每一代聚 合物的分子量与理论值基本一致，说明颈基-烯点击化 学和 CuAAC 点击反应相结合能快速有效的制备树枝状 聚合物.

\section{5 .2 超支化聚合物的制备}

超支化聚合物的制备过程由支臂原料 $\mathrm{A}_{x} \mathrm{~B}_{y}$ 单体 $(y \geqslant 2)$ 相互反应而成, A, B 是具有反应活性的官能团, 链增长发生在不同官能团之间. 颈基点击反应的一系列 优点在超支化聚合物制备领域有广泛的前景.

Balasubramanian 等 ${ }^{[123]}$ 报道了在有机溶剂中利用颈 基一烯点击化学制备超支化间苯二酚纳米微胶囊, 以含 有颈基和双键的双官能团 $\mathrm{A}_{4} \mathrm{~B}_{4}$ 聚合单体杯芳烃类似物 15(如图 20)在氯仿、二氯甲烷、乙酸乙酯和四氢呋喃溶 剂中紫外光引发聚合形成纳米胶囊, DLS 和 SEM 结果 显示不同的溶剂对超支化纳米胶囊的形成有重要影响.

統基-炔点击反应机理表明每个参键官能团可以与 两个颈基官能团发生点击反应，制备的超支化聚合物支 化度较颈基一烯点击反应形成聚合物的支化度快速增大. 

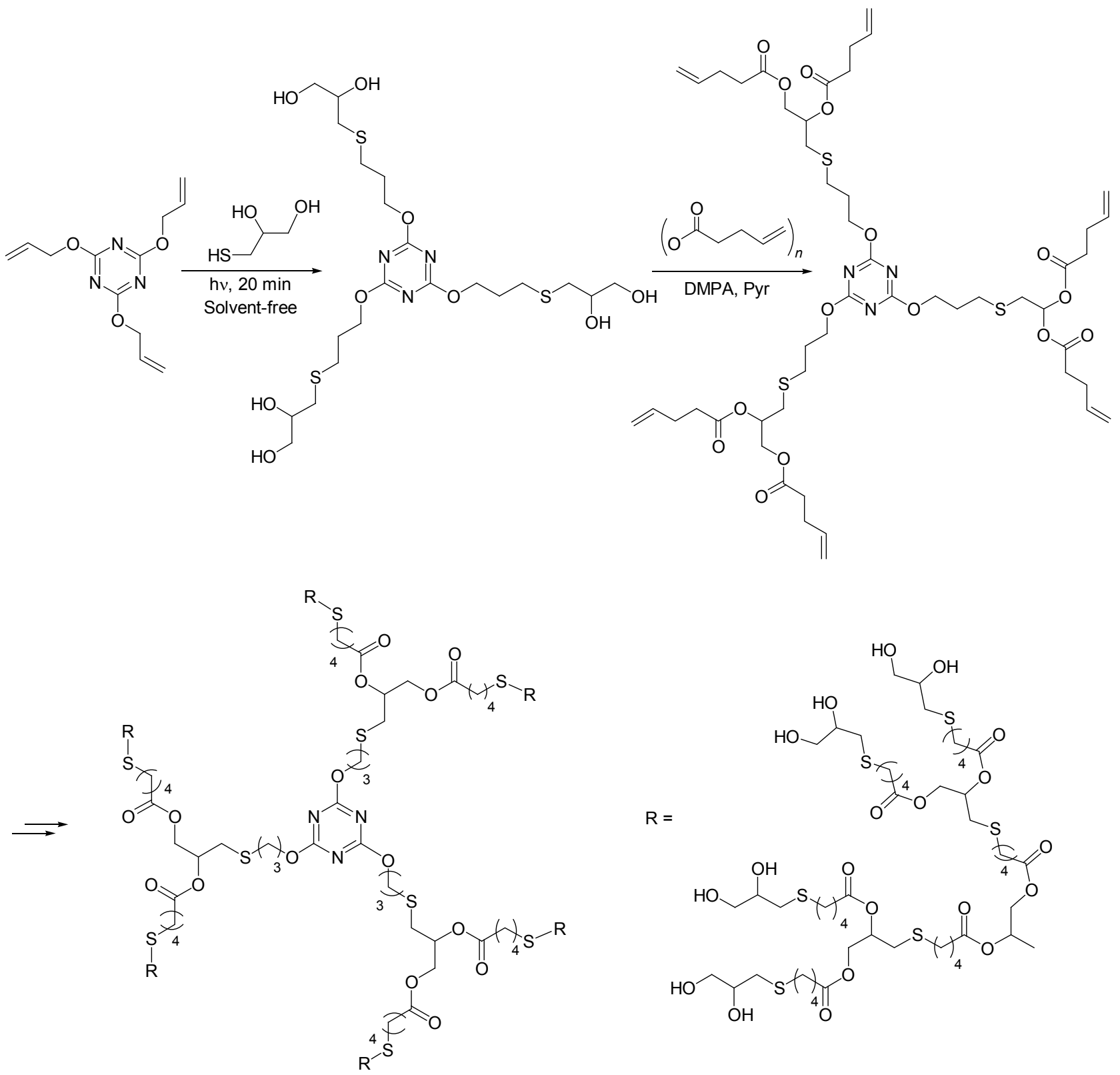

图 17 統基-烯点击反应合成超支化聚合物

Figure 17 Synthesis of hyperbranched polymer via thiol-ene click chemistry

Konkolewicz 等 ${ }^{[124,125]}$ 报道了小分子的支臂单体 $\mathbf{1 7}$ 和大 分子单体 18(如图 21)光聚合制备超支化聚合物的过程, 两种支臂单体在光引发剂安息香二甲醚存在下紫外光 引发聚合, 支化度较一般的 $\mathrm{AB}_{2}$ 单体高, 单体 17 和单体 18 相比具有较高的反应速率, 反应产物中存在大量的 炔基，与 $\mathrm{CuAAC}$ 反应相结合制备功能化聚合物成为可 能.

颈基点击化学对光和热有较高的敏感性, 因此制备 含有颈基和双键或炔基的 $\mathrm{A}_{x} \mathrm{~B}_{y}$ 单体仍存在一定困难, 将是今后一段时间内颈基点击化学制备超支化聚合物
研究的重点.

\section{3 结语及展望}

综上所述, 颈基点击化学具备 CuAAC 和 DielsAlder 反应的反应条件温和、产率高、选择性好和产物 容易分离等特点, 充分的将光引发过程的优点和传统的 点击反应的优点相结合，在特定的区域和官反应，具有 高度的选择性和广泛的适应性，能广泛的应用于聚合物 功能化、大分子构建、材料的设计和合成. 


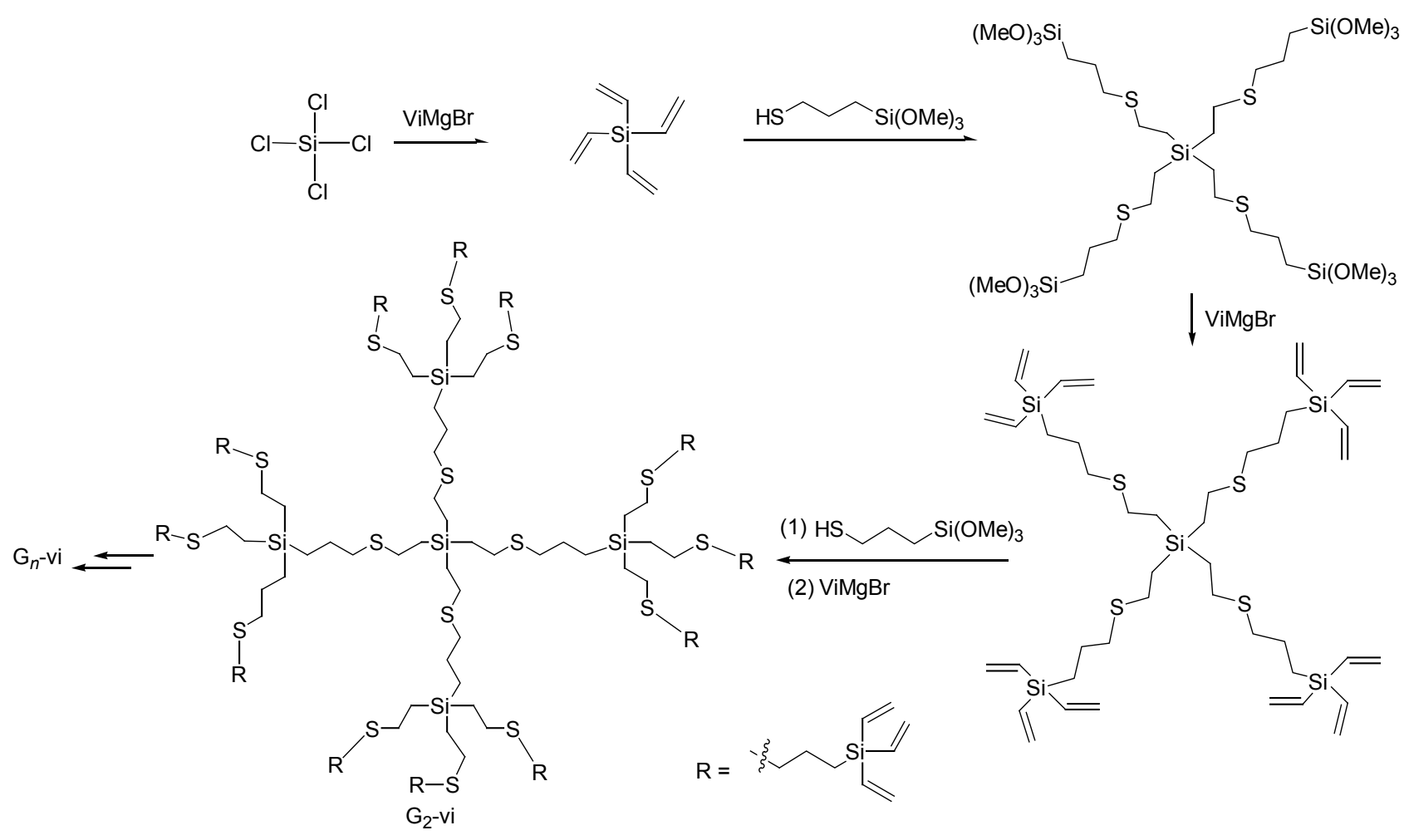

图 18 颈基-烯点击化学合成含硅树枝状聚合物

Figure 18 Synthesis of dendritic polymer containing silicon via thiol-ene click chemistry

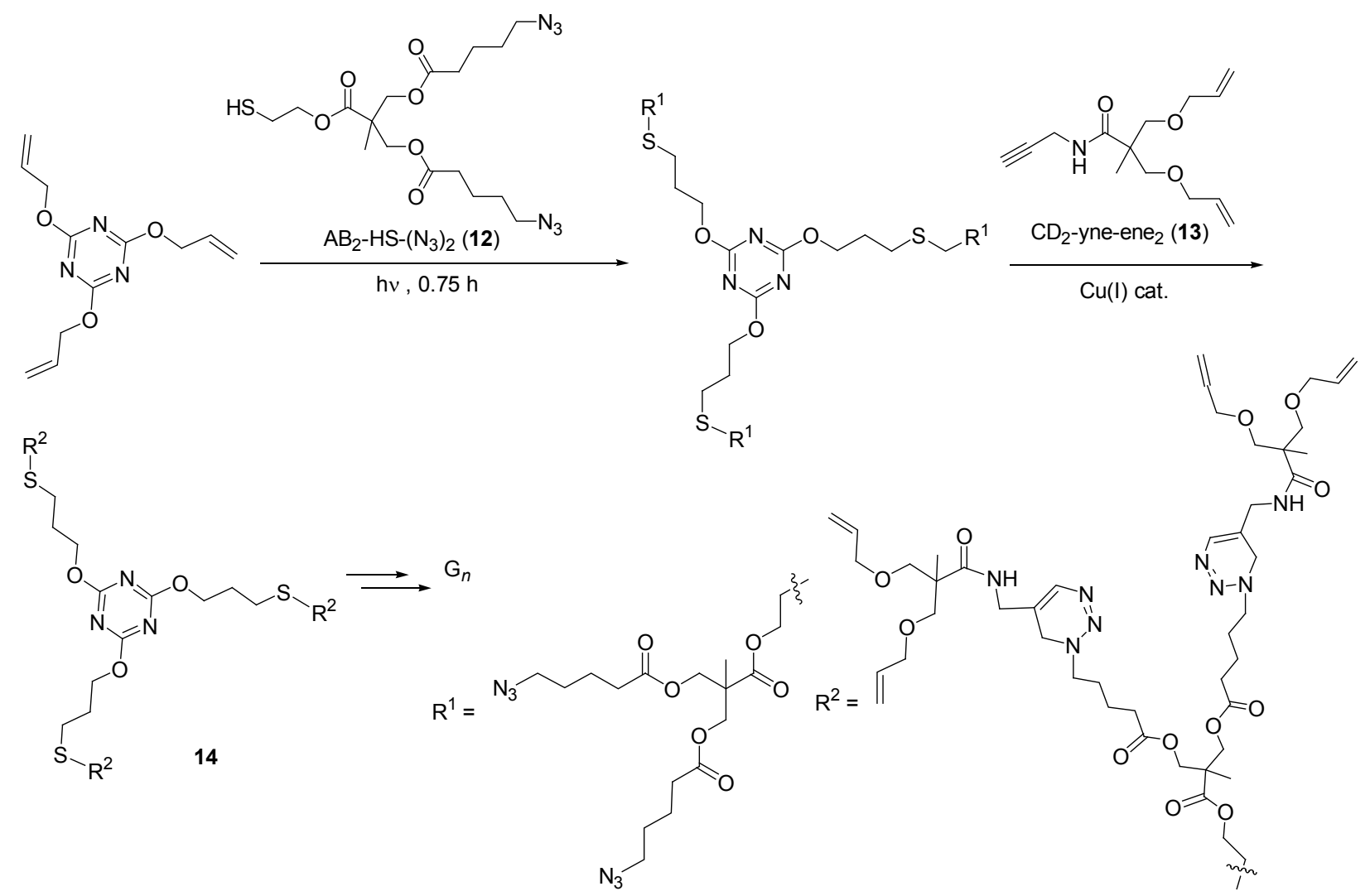

图 19 Thiol-ene 和 CuAAC 点击反应合成树枝状大分子

Figure 19 Synthesis of dendritic polymer facilitated with thiol-ene and $\mathrm{Cu}(\mathrm{I})$-catalyzed azide-alkyne cycloaddition click chemistry 


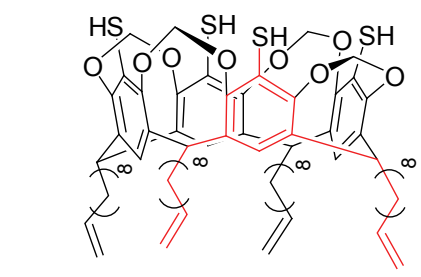

Resorcinarene tetraalkene tetrathiol $\left(\mathrm{A}_{4} \mathrm{~B}_{4}\right)$

15

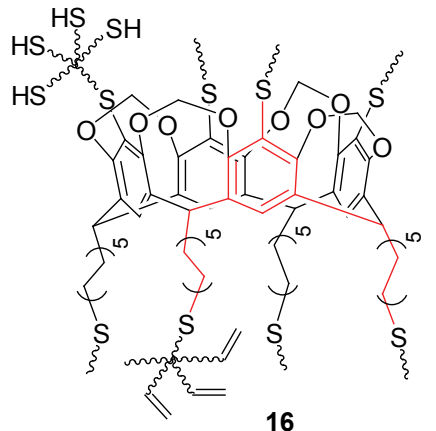

Hyperbranched resorcinarene tetraalkene tetrathiol

图 20 聚合单体和超支化聚合物

Figure 20 Acronym of monomer and hyperbranched polymer via thiol-ene click chemistry<smiles>C#CCOC(=O)CCS</smiles>

17<smiles>C#CCOC(=O)C(C)CC(C)c1ccccc1</smiles>

18

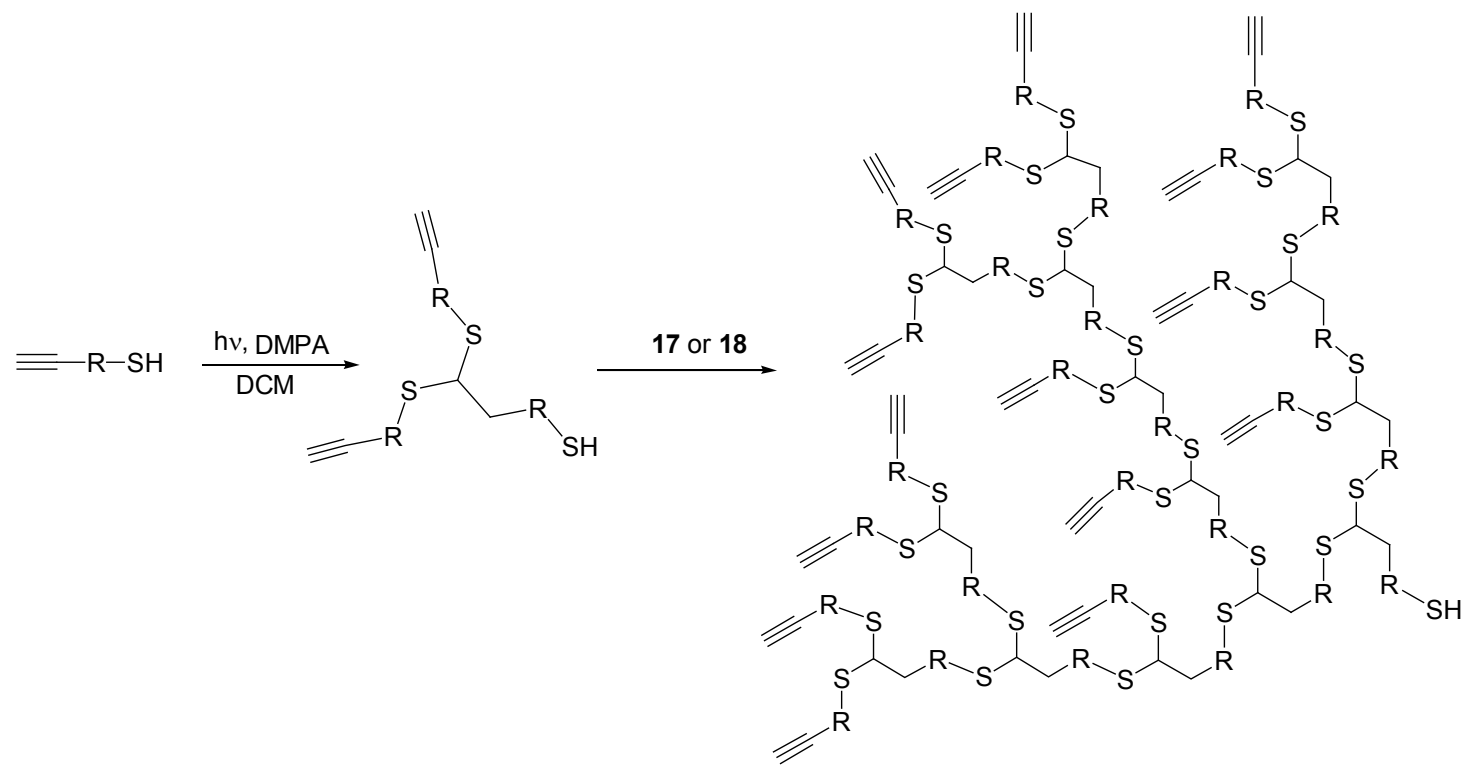

图 21 含巯基和炔基单体合成超支化聚合物示意图

Figure 21 Polymerzation of molecules bearing an alkyne and a thiol to hyperbranched polymer

颈基点击化学作为一种合成材料的新方法, 受到各 国研究人员的高度重视, 目前该反应的应用研究在国内 外开展的如火如茶, 但是颈基点击化学目前的理论和应 用研究存在以下几个问题: (1)自然界和人工合成的双键 单体或聚合物种类多样, 可设计性好, 且合成方法已经 发展了若干年, 但是颈基化合物合成较为复杂且结构种 类不足, 影响了颈基点击化学的应用范围, 开发种类和 官能度不同的颈基化合物应成为以后该领域基础研究 的重点; (2)統基点击化学光交联网络聚合物作为一种巨 大潜力的新型光学材料在如全息聚合物分散液晶和光
至刻蚀等领域方面应开展更多及深入的应用研究; (3)颈 基化合物和双键或参键单体的类型及分子结构对交联 网络材料性能的影响目前的研究不足, 缺乏颈基化合物 和双键单体分子结构和材料性能之间的关系, 是交联网 络制备的研究重点; (4)目前该颈基点击化学的领域的研 究仍处在实验研究阶段, 尚未实现大规模实用产业化或 工业化.

随着点击化学在药物合成及缓释载体材料、聚合物 电解质及光电功能材料领域的深入发展，颈基点击反应 的生物相容性和环境友好性，必将在未来新材料的制备 
过程中必将占据重要地位.

\section{References}

[1] Kolb, C. H.; Finn, M. G.; Sharpless, K. B. Angew. Chem., Int. Ed. 2001, 40, 2004.

[2] Chen, L.; Shi, N. E.; Qian, Y.; Xie, L.-H.; Fan, Q.-L.; Huang, W. Prog. Chem. 2010, 22, 406 (in Chinese).

(陈琳, 石乃恩, 钱妍, 解令海, 范曲立, 黄维, 化学进展, 2010, 22, 406.)

[3] Wang, C.; Wang, N.; Zhou, W.; Shen, Y. M.; Zhang, L. Prog. Chem. 2010, 22, 1591 (in Chinese). (王成, 王妮, 周伟, 沈玉梅, 张岗, 化学进展, 2010, 22, 1591.)

[4] Ates, S.; Durmaz, Y. Y.; Torun, L.; Yagci, Y. J. Macromol. Sci. A 2010, 47, 809 .

[5] Lau, K. N.; Chow, H. F.; Chan, M. C.; Wong, K. W. Angew. Chem., Int. Ed. 2008, 47, 6912.

[6] Tonga, M.; Cengiz, N.; Kose, M. M.; Dede, T.; Sanyal, A. J. Polym. Sci., Polym. Chem. 2010, 48, 410

[7] Semsarilar, M.; Ladmiral, V.; Perrier, S. Macromolecules 2010, 43, 1438.

[8] Nilsson, C.; Simpson, N.; Malkoch, M.; Johansson, M.; Malmström, E. J. Polym. Sci., Polym. Chem. 2008, 46, 1339.

[9] Tan, M. Q.; Wu, X. M.; Jeong, E. K. Mol. Pharm. 2010, 7, 936.

[10] Cunningham, C. W.; Mukhopadhyay, A.; Lushington, G. H. Mol. Pharm. 2010, 7, 1301

[11] Glaser, M.; Arstad, E. Bioconjugate Chem. 2007, 18, 989.

[12] Speers, A. E.; Adam, G. C.; Cravatt, B. F. J. Am. Chem. Soc. 2003, 125,4686

[13] Vila, A.; Tallman, K. A.; Jacobs, A. T.; Liebler, D. C.; Porter, N. A.; Marnett, L. J. Chem. Res. Toxicol. 2008, 21, 432.

[14] Li, M.; De, P.; Gondi, S. R.; Sumerlin, B. S. Macromol. Rapid Commun. 2008, 29, 1172.

[15] Lewis, W. G.; Green, L. G.; Grynszpan, F.; Radic, Z.; Carlier, P. R.; Taylor, P.; Finn, M. G.; Sharpless, K. B. Angew. Chem., Int. Ed. 2002, 41, 1053.

[16] Gopin, A.; Ebner, S.; Attali, B.; Shabat, D. Bioconjugate Chem. 2006, 17, 1432 .

[17] Lee, L. V.; Mitchell, M. L.; Huang, S. J.; Fokin, V. V.; Sharpless, K. B.; Wong, C. H. J. Am. Chem. Soc. 2003, 125, 9588.

[18] Baskina, J. M.; Bertozzi, C. R. QSAR Comb. Sci. 2007, 26, 1211.

[19] Lin, C.-C.; Raza, A.; Shih, H. Biomaterials 2011, 32, 9685.

[20] Baskin, J. M.; Prescher, J. A.; Laughlin, S. T.; Agard, N. J.; Chang, P. V.; Miller, I. A.; Codelli, J. A.; Bertozzi, C. R. Proc. Natl. Acad. Sci. 2007, 16793.

[21] Oria, L.; Aguado, R.; Pomposo, J. A.; Colmenero, J. Adv. Mater. 2010, 22, 3038.

[22] O'reilly, R. K.; Joralemon, M. J.; Hawker, C. J.; Wooley, K. L. J. Polym. Sci., Part A: Polym. Chem. 2006, 44, 5203

[23] Devaraj, N. K.; Keliher, E. J.; Thurber, G. M.; Nahrendorf, M.; Weissleder, R. Bioconjugate Chem. 2009, 20, 397.

[24] Huynh, V. T.; Chen, G.-J.; Souza, P.; Stenzel, H. M. Biomacromolecules 2011, 12, 1738.

[25] Such, G. K.; Tjipto, E.; Postma, A.; Johnston, A. P. R.; Caruso, F. Nano Lett. 2007, 7, 1706.

[26] Suksiriworapong, J.; Sripha, K.; Kreuter, J.; Junyaprasert, V. B. Bioconjugate Chem. 2011, 22, 582

[27] Breed, D.; Thibault, R.; Xie, F.; Wang, Q.; Hawker, C. J.; Pine, D. J. Langmuir 2009, 25, 4370.

[28] Cavalieri, F.; Postma, A.; Lee, L.; Caruso, F. ACS Nano. 2009, 3, 234.
[29] Schreck, M. K.; Leung, D.; Bowman, N. C. Macromolecules 2011, 44, 7520.

[30] Fu, Y.; Liu, Y.; Fu, X.; Zou, L.; Li, H.; Li, M.; Chen, X.; Qin, J. Chin. J. Chem. 2010, 28, 2226

[31] Bhagat, D. S.; Chatterjee, J.; Chen, B.-H.; Stiegman, A. E. Macromolecules, 2012, 45, 1174.

[32] Tucker-Schwartz, K. A.; Farrell, R. A.; Garrell, R. L. J. Am. Chem. Soc. 2011, 133, 11026

[33] Jonkheijm, P.; Weinrich, D.; Köhn, M.; Engelkamp, H.; Christianen, P. C. M.; Kuhlmann, J., Maan, J. C.; Nüsse, D.; Schroeder, H.; Wacker, R.; Breinbauer, R.; Niemeyer, C. M.; Waldmann, H. Nat. Mater. 2009, 8, 659.

[34] Jacobine, F. A. Radiation Curing in Polymer Science and Technology: Polymerisation Mechanisms, Washingtion, D. C., 1993, Chapter 7 , p. 220.

[35] Hoyle, C. E.; Lee, T.-Y.; Roper, T. J. Polym. Sci., Polym. Chem. 2004, 42, 5301.

[36] Hoyle, C. E.; Bowman, C. N. Angew. Chem., Int. Ed. 2010, 49, 1540

[37] Hoyle, C. E.; Lowe, A. B.; Bowman, C. N. Chem. Soc. Rev. 2010, $39,1355$.

[38] Posner T. Ber. Dtsh. Chem. Ges. 1905, 38, 646.

[39] Kharasch, M. S.; Read, A. T.; Mayo F. R. Chem. Ind. 1938, 57, 752.

[40] Hoyle, C. E.; Lee, T.-Y; Roper, T. J. Polym. Sci., Polym. Chem. 2004, 42, 5301.

[41] Li, Q.; Zhou, H.; Hoyle, C. E. Polymer 2009, 50, 2237.

[42] Shin, J.; Sergei, N.; Phillips, J. P.; Hoyle, C. E. Polymer 2009, 50, 6281.

[43] Liu, Q.; Zhang, Q.-Y.; Zhou, J.; Zhang, H. P. Mater. Rev. 2011, 10, 140 (in Chinese)

(刘清, 张秋禹, 周健, 张和鹏, 材料导报, 2011, 10, 140.)

[44] Zhou, J.; Zhang, Q.-Y.; Zhang, H.-P.; Chen, S.-J.; Liu, Q. J. Funct. Mater. 2012, 43, 1494 (in Chinese).

(周健, 张秋禹, 张和鹏, 陈少杰, 刘清, 功能材料, 2012, 43, 1494.)

[45] Finzi; Gazz, C. Chim. Ital. 1930, 60, 798.

[46] Kohler, E. P.; Potter, H. J. Am. Chem. Soc. 1935, 57, 1316.

[47] Lowe, A. B.; Hoyle, C. E.; Bowman, C. N. J. Mater. Chem. 2010, 20,4745 .

[48] Chan, J. W.; Hoyle, C. E.; Lowe, A. B. J. Am. Chem. Soc. 2009, 131,5751

[49] Hensarling, R. M.; Doughty, V. A.; Chan, J. W.; Patton, D. L. J. Am. Chem. Soc. 2009, 131, 14673.

[50] Fairbanks, B. D.; Scott, T. F.; Kloxin, C. J.; Anseth, K. S.; Bowman, C. N. Macromolecules 2009, 42, 211

[51] Fairbanks, B. D.; Sims, E. A.; Anseth, K. S.; Bowman, C. N. Macromolecules 2010, 43, 4113.

[52] Chan, J. W.; Shin, J.; Hoyle, C. E.; Bowman, C. N.; Lowe, A. B. Macromolecules 2010, 43, 4937.

[53] Yu, B.; Chan, J. W.; Hoyle, C. E.; Lowe, A. B. J. Polym. Sci., Polym. Chem. 2009, 47, 3544.

[54] Naik, S. S.; Chan, J. W.; Comer, C.; Hoyle, C. E.; Savin, D. A. Polym. Chem. 2011, 2, 303.

[55] Chan, J. W.; Zhou, H.; Hoyle, C. E.; Lowe, A. B. Chem. Mater. 2009, 21, 1579.

[56] Lee, T. Y.; Roper, T. M.; Guymon, C. A.; Hoyle, C. E. Macromolecules 2004, 37, 3606.

[57] Cramer, N. B.; Reddy, S. K.; O'Brien, A. K.; Bowman, C. N. Macromolecules 2003, 36, 7964.

[58] Reddy, S. K.; Cramer, N. B.; Bowman, C. N. Macromolecules 
2006, 39, 3673.

[59] Reddy, S. K.; Cramer, N. B.; Bowman, C. N. Macromolecules 2006, 39, 3681.

[60] Fairbanks, B. D.; Scott, T. F.; Kloxin, C. J.; Anseth, K. S.; Bowman, C. N. Macromolecules 2010, 43, 4937

[61] Fairbanks, B. D.; Sims, E. A.; Anseth, K. S.; Bowman, C. N. Macromolecules 2010, 43, 4113.

[62] Fairbanks, B. D.; Scott, T. F.; Kloxin, C. J.; Anseth, K. S.; Bowman, C. N. Macromolecules 2009, 42, 211.

[63] Korthals, B.; Morant, M. M. C.; Schmid, M.; Mecking, S.; Macromolecules 2010, 43, 8071 .

[64] Prasath, R. A.; Gokmen, M. T.; Espeel, P.; Prez, F. E. D. Polym. Chem. 2010, 1, 685 .

[65] van der Ende, A. E.; Harrell, J.; Sathiyakumar, V.; Meschievitz, M.; Katz, J.; Adcock, K.; Harth, E. Macromolecules 2010, 43, 5665.

[66] van der Ende, A. E.; Croce, T.; Hamilton, S.; Sathiyakumar, V.; Harth, E. Soft Matter 2009, 5, 1417.

[67] Iskin, B.; Yilmaz, G.; Yagci, Y. J. Polym. Sci., Polym. Chem. 2011, 49, 2417.

[68] Wang, W.-J.; Li, T.; Yu, T.; Zhu, F.-M. Macromolecules 2008, 41, 9750.

[69] Li, J.; He, W.-D.; He, N.; Han, S.-C.; Sun, X.-L, Li, L.-Y.; Zhang, B.-Y. J. Polym. Sci., Polym. Chem. 2009, 47, 1450

[70] Li, J.; He, W.-D.; Han, S.-C.; Sun, X.-L.; Li, L.-Y.; Zhan, B.-Y. J. Polym. Sci., Polym. Chem. 2009, 47, 786.

[71] Dong, Y. X.; Saeed, A. O.; Hassan, W.; Keigher, C.; Zheng, Y.; Tai, H. Y.; Pandit, A.; Wang, W. X. Macromol. Rapid Commun. 2012, 33, 120 .

[72] Chen, G.-J.; Amajjahe, S.; Stenzel, M. Chem. Commun. 2009, 1198.

[73] Wong, L.-J.; Sevimli, S.; Zareie, H. M.; Davis, T. P.; Bulmus, V. Macromolecules 2010, 43,5365.

[74] Wong, L.-J.; Kavallaris, M.; Bulmus, V. Polym. Chem. 2011, 2, 385.

[75] Mantovani, G.; Ladmiral, V.; Tao, L.; Haddleton, D. M. Chem. Commun. 2005, 2089

[76] Tsarevsky, N. V.; Sumerlin, B. S.; Matyjaszewski, K. Macromolecules 2005, 38, 3558 .

[77] Malkoch, M.; Thibault, R. J.; Drockenmuller, E.; Messerschmidt, M.; Voit, B.; Russell, T. P.; Hawker, C. J. J. Am. Chem. Soc. 2005, $127,14942$.

[78] O-Reilly, R. K.; Joralemon, M. J.; Hawker, C. J.; Wooley, K. L. Chem. Eur. J. 2006, 12, 6776.

[79] Thomsen, A. D.; Malmström, E.; Hvilsted, S. J. Polym. Sci., Polym. Chem. 2006, 44, 6360 .

[80] Parrish, B.; Breitenkamp, R. B.; Emrick, T. J. Am. Chem. Soc. 2005, 127, 7404.

[81] Li, Z.-A.; Zeng, Q.; Li, Z.; Zhu, Z.-C.; Li, Q.-Q.; Ye, C.; Di, C.-A.; Liu, Y.-Q.; Qin, J.-G. Macromolecules 2006, 39, 8544.

[82] Acik, G.; Kahveci, M. U.; Yagci, Y. Macromolecules 2010, 43, 9198.

[83] Temel, G.; Aydogan, B.; Arsu, N. Y. Macromolecules 2009, 42, 6098 .

[84] Zhang, S.-H.; Zhu, H.-W.; Zhang, M.-Z.; Ni, P.-H. Acta Chim. Sinica 2011, 69, 2166 (in Chinese). (张世华, 朱汇雯, 张明祖, 倪沛红, 化学学报, 2011, 69, 2166.)

[85] Hoyle, C. E.; Bowman, C. N. Angew. Chem., Int. Ed. 2010, 49, 1540.

[86] Lowe, A. B. Polym. Chem. 2010, 1, 17.

[87] Hoyle, C. E.; Lowe, A. B.; Bowman, C. N. Chem. Soc. Rev. 2010, $39,1355$.
[88] Huynh, T.; Chen, G.-J.; Souza, P.; Stenzel, M. H. Biomacromolecules 2011, 12, 1738 .

[89] Stamenovi, M.; Espeel, P.; Camp, W. V.; Du, F. E.; Konkolewicz, P.; Poon, C. K.; Gray-Weale, A.; Perrier, S. Chem. Commun. 2011, $47,239$.

[90] Jones, T. W.; Gibson, M. I.; Mantovani, G.; Haddletona, D. M. Polym. Chem. 2011, 2, 57.

[91] Chen, J.-C.; Liu, M.-Z.; Chen, C.; Gong, H.-H.; Gao, C.-M. ACS Appl. Mater. Interfaces 2011, 3, 3215.

[92] Nicolay, R. Macromolecules 2012, 45, 821.

[93] Roth, P. J.; Boyer, C.; Lowe, A. B.; Davis, T. P. Macromol. Rapid Commun. 2011, 32, 1123.

[94] Abdellatif, M. M.; Nomura, K. ACS Macro Lett. 2012, 1, 423.

[95] Huang, C.; Neoh, K. G.; Kang, E.-T. Langmiur 2012, 28, 563.

[96] Uygun, M.; Tasdelen, M. A.; Yagci, Y. Macromol. Chem. Phys. 2010, 211, 103.

[97] Nurmi, L.; Lindqvist, J.; Randev, R.; Syrett, J.; Haddleton, D. M. Chem. Commun. 2009, 2727.

[98] Soeriyadi, A. H.; Li, G.-Z.; Slavin, S.; Slavin, S.; Jones, M. W.; Amos, C. M.; Becer, C. R.; Whittaker, M. R.; Haddleton, D. M.; Boyer, C.; Davis, T. P. Polym. Chem. 2011, 2, 815.

[99] Goldmann, A. S.; Walther, A.; Nebhani, L.; Joso, R.; Ernst, D.; Loos, K.; Barner-Kowollik, C.; Barner, L.; Müller, A. H. E. Macromolecules 2009, 42, 3707.

[100] Li, G.-L.; Wan, D.; Neoh, G. K.; Kang, E. T. Macromolecules 2010, 43, 10275 .

[101] Boyer, C.; Soeriyadi, A. H.; Roth, P. J.; Whittaker, M. R.; Davis, T. P. Chem. Commun. 2011, 47, 1318 .

[102] Chan, J. W.; Yu, B.; Hoyle, C. E.; Lowe, A. B. Chem. Commun. 2008, 4959.

[103] Yu, B.; Chan, J. W.; Hoyle, C. E.; Lowe, A. B. J. Polym. Sci., Polym. Chem. 2009, 47, 3544

[104] Li, M.; De, P.; Gondi, S. R.; Sumerlin, B. S. J. Polym. Sci., Polym. Chem. 2008, 46, 5093.

[105] Cyrille, B.; Anthony, G.; Thomas, P. D.; Bulmus, V. J. Polym. Sci., Polym. Chem. 2009, 47, 3773.

[106] Liu, W.; Dong, C. M. Macromolecules 2010, 43, 8447.

[107] Zheng, H.; Li, Y.-L.; Zhou, C. J. Chem. Eur. J. 2011, 17, 2160.

[108] Zhou, W.-D.; Zheng, H.-Y.; Li, Y.-J.; Li, Y.-L. Org. Lett. 2010, 12, 4078.

[109] Campos, L. M.; Killops, K. L.; Sakai, R.; Paulusse, J. M. J.; Damiron, D.; Drockenmuller, E.; Messmore, B. W.; Hawker, C. J. Macromolecules 2008, 41, 7063.

[110] Heggli, M.; Tirelli, N.; Zisch, A.; Hubbell, J. A. Bioconjugate Chem. 2003, 14, 967.

[111] Rutledge, R. D.; Warner, C. L.; Pittman, J. W.; Engelhard, M.; Chouyyok, W.; Warner, M. G. Langmuir 2010, 26, 12285.

[112] Berkel, K. Y. V.; Hawker, C. J. J. Polym. Sci., Polym. Chem. 2010, $48,1594$.

[113] Salinas, C. N.; Anseth, K. S. Macromolecules 2008, 41, 6019.

[114] Li, Y.-H.; Wang, D.; Buriak, J. M. Langmuir 2010, 26, 1232.

[115] Khire, V. S.; Lee, T. Y.; Bowman, C. N. Macromolecules 2008, 41, 7440 .

[116] Lee, T. Y.; Bowman, C. N. Polymer 2006, 47, 6057.

[117] Killops, K. L.; Campos, L. M.; Hawker, C. J. J. Am. Chem. Soc. 2008, 130, 5062 .

[118] Montañez, M. I.; Campos, L. M.; Antoni, P.; Hed, Y.; Walter, M. V.; Krull, B. T.; Khan, A.; Hult, A.; Hawker, C. J.; Malkoch, M. Macromolecules 2010, 43, 6004.

[119] Rim, C.; Son, D. Y. Tetrahedron Lett. 2009, 50, 4161.

[120] Rissing, C.; Son, D. Y. Organometallics 2008, 27, 5394. 
[121] Rissing, C.; Son, D. Y. Organometallics 2009, 28, 3167

[122] Antoni, P.; Robb, M. J.; Campos, L.; Montanez, M.; Hult, A.; Malmström, E.; Michael, M.; Hawker, C. J. Macromolecules 2010, 43, 6625

[123] Balasubramanian, R.; Kalaitzis, Z. M.; Cao, W. J. Mater. Chem.
2010, 20, 6539.

[124] Konkolewicz, D.; Gray-Weale, A.; Perrier, S. J. Am. Chem. Soc. 2009, 131, 18075.

[125] Konkolewicz, D.; Poon, C. K.; Gray-Weale, A.; Perrier, S. Chem. Commun. 2011, 47, 239. 